\title{
Higher-Dimensional Unified Theories with Fuzzy Extra Dimensions ${ }^{\star}$
}

\author{
Athanasios CHATZISTAVRAKIDIS ${ }^{\dagger}$ and George ZOUPANOS $\ddagger$ \\ † Institute of Nuclear Physics, NCSR Demokritos, GR-15310 Athens, Greece \\ E-mail: cthan@mail.ntua.gr \\ $\ddagger$ Physics Department, National Technical University of Athens, \\ GR-15780 Zografou Campus, Athens, Greece \\ E-mail: george.zoupanos@cern.ch
}

Received May 06, 2010, in final form July 22, 2010; Published online August 12, 2010

doi:10.3842/SIGMA.2010.063

\begin{abstract}
Theories defined in higher than four dimensions have been used in various frameworks and have a long and interesting history. Here we review certain attempts, developed over the last years, towards the construction of unified particle physics models in the context of higher-dimensional gauge theories with non-commutative extra dimensions. These ideas have been developed in two complementary ways, namely (i) starting with a higher-dimensional gauge theory and dimensionally reducing it to four dimensions over fuzzy internal spaces and (ii) starting with a four-dimensional, renormalizable gauge theory and dynamically generating fuzzy extra dimensions. We describe the above approaches and moreover we discuss the inclusion of fermions and the construction of realistic chiral theories in this context.
\end{abstract}

Key words: fuzzy extra dimensions; unified gauge theories; symmetry breaking

2010 Mathematics Subject Classification: 70S15

\section{Contents}

1 Introduction $\quad 2$

2 Fuzzy spaces and dimensional reduction $\quad 4$

2.1 The fuzzy sphere . . . . . . . . . . . . . . . . . . 4

2.1.1 Gauge theory on the fuzzy sphere . . . . . . . . . . . . 7

2.2 Dimensional reduction and gauge symmetry enhancement . . . . . . . . . . 8

2.3 Non-trivial dimensional reduction over fuzzy extra dimensions . . . . . . . . . . 9

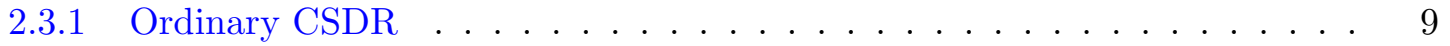

2.3 .2 Fuzzy CSDR . . . . . . . . . . . . . . . . . . 11

2.3.3 Solving the CSDR constraints for the fuzzy sphere . . . . . . . . . . . . . 12

2.4 The problem of chirality in fuzzy CSDR . . . . . . . . . . . . . 13

3 Dynamical generation of fuzzy extra dimensions $\quad 16$

3.1 The four dimensional action . . . . . . . . . . . . . . 16

3.2 Emergence of extra dimensions and the fuzzy sphere . . . . . . . . . . . . 17

3.3 Inclusion of fermions . . . . . . . . . . . . . . . . . . . . . . . . 19

3.3.1 Fermions on $M^{4} \times S^{2}$ and $M^{4} \times S_{N}^{2} \ldots \ldots \ldots \ldots \ldots \ldots$

3.3.2 The spectrum of $\not_{(2)}$ in a type I vacuum . . . . . . . . . 20

\footnotetext{
*This paper is a contribution to the Special Issue "Noncommutative Spaces and Fields". The full collection is available at http://www.emis.de/journals/SIGMA/noncommutative.html
} 
3.3.3 The spectrum of $D_{(2)}$ in a type II vacuum . . . . . . . . . . . . 21

3.4 Dynamical generation of fuzzy $S^{2} \times S^{2}$ and mirror fermions . . . . . . . . . 21

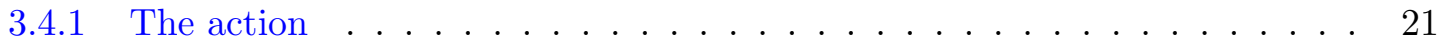

3.4.2 Type I vacuum and fuzzy $S^{2} \times S^{2} \ldots \ldots \ldots \ldots \ldots 24$

3.4.3 Operators on $S_{L}^{2} \times S_{R}^{2} \ldots \ldots \ldots \ldots \ldots \ldots \ldots \ldots$

3.4 .4 Type II vacuum and the zero-modes . . . . . . . . . . . . . 26

4 Orbifolds, fuzzy extra dimensions and chiral models $\quad 28$

$4.1 \mathcal{N}=4 \mathrm{SYM}$ and $\mathbb{Z}_{3}$ orbifolds . . . . . . . . . . . . . . . . . 28

4.2 Twisted fuzzy spheres . . . . . . . . . . . . . . . . . . . 30

4.3 Dynamical generation of twisted fuzzy spheres . . . . . . . . . . . . . . 32

4.4 Chiral models from the fuzzy orbifold . . . . . . . . . . . . . . . . . . . . . . . . . .

4.4.1 A $S U(4)_{c} \times S U(2)_{L} \times S U(2)_{R}$ model . . . . . . . . . . . . 34

4.4.2 A $S U(4)_{c} \times S U(4)_{L} \times S U(4)_{R}$ model . . . . . . . . . . . . 35

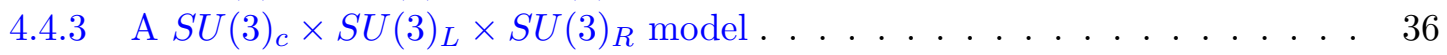

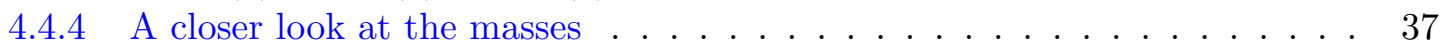

4.5 Fuzzy breaking for $S U(3)^{3} \ldots \ldots \ldots \ldots \ldots \ldots \ldots$

5 Discussion and conclusions $\quad 40$

$\begin{array}{ll}\text { A Clifford algebra conventions } & 42\end{array}$

$\begin{array}{ll}\text { References } & 42\end{array}$

\section{Introduction}

The unification of the fundamental interactions has always been one of the main goals of theoretical physics. Several approaches have been employed in order to achieve this goal, one of the most exciting ones being the proposal that extra dimensions may exist in nature. The most serious support on the existence of extra dimensions came from superstring theories [1], which at present are the best candidates for a unified description of all fundamental interactions, including gravity and moreover they can be consistently defined only in higher dimensions. Among superstring theories the heterotic string [2] has always been considered as the most promising version in the prospect to find contact with low-energy physics studied in accelerators, mainly due to the presence of the ten-dimensional $\mathcal{N}=1$ gauge sector. Upon compactification of the ten-dimensional space-time and subsequent dimensional reduction the initial $E_{8} \times E_{8}$ gauge theory can break to phenomenologically interesting Grand Unified Theories (GUTs), where the Standard Model (SM) could in principle be accommodated [2]. Dimensional reduction of higher-dimensional gauge theories had been studied few years earlier than the discovery of the heterotic superstring with pioneer studies the Forgacs-Manton Coset Space Dimensional Reduction (CSDR) $[3,4,5]$ and the Scherk-Schwarz group manifold reduction [6]. In these frameworks gauge-Higgs unification is achieved in higher dimensions, since the four-dimensional gauge and Higgs fields are simply the surviving components of the gauge fields of a pure gauge theory defined in higher dimensions. Moreover in the CSDR the addition of fermions in the higherdimensional gauge theory leads naturally to Yukawa couplings in four dimensions. A major achievement in this direction is the possibility to obtain chiral theories in four dimensions [7].

On the other hand, non-commutative geometry offers another framework aiming to describe physics at the Planck scale $[8,9]$. In the spirit of non-commutative geometry also particle models with non-commutative gauge theory were explored [10] (see also [11]), [12, 13]. It is worth stressing the observation that a natural realization of non-commutativity of space appears 
in the string theory context of $D$-branes in the presence of a constant antisymmetric field [14], which not only brought together the two approaches but they can be considered complementary. Another interesting development in the non-commutative framework was the work of Seiberg and Witten [15], where a map between the non-commutative and commutative gauge theories has been described. Based on that and related subsequent developments [16, 17] a non-commutative version of the SM has been constructed [18]. These non-commutative models represent interesting generalizations of the SM and hint at possible new physics. However they do not address the usual problem of the SM, the presence of a plethora of free parameters mostly related to the ad hoc introduction of the Higgs and Yukawa sectors in the theory.

According to the above discussion it is natural to investigate higher-dimensional gauge theories and their dimensional reduction in four dimensions. Our aim is to provide an up to-date overview of certain attempts in this direction, developed over the last years. The development of these ideas has followed two complementary ways, namely (i) the dimensional reduction of a higher-dimensional gauge theory over fuzzy internal spaces [19] and (ii) the dynamical generation of fuzzy extra dimensions within a four-dimensional and renormalizable gauge theory $[20,21,22,23]$.

More specifically, the paper is organized as follows. In Section 2 we present a study of the CSDR in the non-commutative context which sets the rules for constructing new particle models that might be phenomenologically interesting. One could study CSDR with the whole parent space $M^{D}$ being non-commutative or with just non-commutative Minkowski space or noncommutative internal space. We specialize here to this last situation and therefore eventually we obtain Lorentz covariant theories on commutative Minkowski space. We further specialize to fuzzy non-commutativity, i.e. to matrix type non-commutativity. Thus, following [19], we consider non-commutative spaces like those studied in $[9,12,13]$ and implementing the CSDR principle on these spaces we obtain the rules for constructing new particle models. In Section 2.1 the fuzzy sphere is introduced and moreover the gauge theory on the fuzzy sphere is discussed. In Section 2.2 a trivial dimensional reduction of a higher-dimensional gauge theory over the fuzzy sphere is performed. In Section 2.3 we discuss the non-trivial dimensional reduction; first the CSDR scheme in the commutative case is briefly reviewed and subsequently it is applied to the case of fuzzy extra dimensions. In Section 2.4 the issue of chirality is discussed within the above context.

In Section 3 we reverse the above approach [20] and examine how a four-dimensional gauge theory dynamically develops fuzzy extra dimensions. In Sections 3.1 and 3.2 we present a simple field-theoretical model which realizes the above ideas. It is defined as a renormalizable $S U(N)$ gauge theory on four-dimensional Minkowski space $M^{4}$, containing three scalars in the adjoint of $S U(N)$ that transform as vectors under an additional global $S O(3)$ symmetry with the most general renormalizable potential. We then show that the model dynamically develops fuzzy extra dimensions, more precisely a fuzzy sphere $S_{N}^{2}$. The appropriate interpretation is therefore as gauge theory on $M^{4} \times S_{N}^{2}$. The low-energy effective action is that of a four-dimensional gauge theory on $M^{4}$, whose gauge group and field content is dynamically determined by compactification and dimensional reduction on the internal sphere $S_{N}^{2}$. An interesting and rich pattern of spontaneous symmetry breaking appears, namely the breaking of the original $S U(N)$ gauge symmetry down to either $S U(n)$ or $S U\left(n_{1}\right) \times S U\left(n_{2}\right) \times U(1)$. The latter case is the generic one, and implies also a monopole flux induced on the fuzzy sphere. Moreover we determine explicitly the tower of massive Kaluza-Klein modes corresponding to the effective geometry, which justifies the interpretation as a compactified higher-dimensional gauge theory. Last but not least, the model is renormalizable. In Sections 3.3 and 3.4 we explore the dynamical generation of a product of two fuzzy spheres [22]. Specifically, we start with the $S U(N)$ Yang-Mills theory in four dimensions, coupled to six scalars and four Majorana spinors, i.e. with the particle spectrum of the $\mathcal{N}=4$ supersymmetric Yang-Mills theory (SYM). Adding 
an explicit $R$-symmetry-breaking potential, thus breaking the $\mathcal{N}=4$ supersymmetry, we reveal stable $M^{4} \times S_{L}^{2} \times S_{R}^{2}$ vacua. In the most interesting case we include magnetic fluxes on the extra-dimensional fuzzy spheres and study the fermion spectrum, in particular the zero modes of the Dirac operator. The outcome of our analysis is that we obtain a mirror model in low energies.

In Section 4 we present a recently developed approach within the above framework, which leads to chiral low-energy models [23]. In particular, $\mathbb{Z}_{3}$ orbifolds of $\mathcal{N}=4$ supersymmetric Yang-Mills theory are discussed and they are subsequently used to dynamically generate fuzzy extra dimensions. The extra dimensions are described by twisted fuzzy spheres, defined in Section 4.2. This framework allows to construct low-energy models with interesting unification groups and a chiral spectrum. In particular, we are led to study three different models based on the gauge groups $S U(4) \times S U(2) \times S U(2), S U(4)^{3}$ and $S U(3)^{3}$ respectively. The spontaneous symmetry breaking of the latter unified gauge group down to the minimal supersymmetric standard model and to the $S U(3) \times U(1)_{e m}$ is subsequently studied within the same framework. Finally, Section 5 contains our conclusions.

\section{Fuzzy spaces and dimensional reduction}

\subsection{The fuzzy sphere}

The fuzzy sphere [24] is a noncommutative manifold which corresponds to a matrix approximation of the ordinary sphere. In order to describe it let us consider the ordinary sphere as a submanifold of the three-dimensional Euclidean space $\mathbb{R}^{3}$. The coordinates of $\mathbb{R}^{3}$ will be denoted as $x_{a}, a=1,2,3$. Then the algebra of functions on the ordinary sphere $S^{2} \subset \mathbb{R}^{3}$ can be generated by the coordinates of $\mathbb{R}^{3}$ modulo the relation

$$
\sum_{a=1}^{3} x_{a} x_{a}=R^{2},
$$

where $R$ is the radius of the sphere. Clearly, the sphere admits the action of a global $S O(3) \sim$ $S U(2)$ isometry group. The generators of $S U(2) \sim S O(3)$ are the three angular momentum operators $L_{a}$,

$$
L_{a}=-i \varepsilon_{a b c} x_{b} \partial_{c},
$$

which in terms of the usual spherical coordinates $\theta$ and $\phi$ become

$$
\begin{aligned}
& L_{1}=i \sin \phi \frac{\partial}{\partial \theta}+i \cos \phi \cot \theta \frac{\partial}{\partial \phi} \\
& L_{2}=-i \cos \phi \frac{\partial}{\partial \theta}+i \sin \phi \cot \theta \frac{\partial}{\partial \phi} \\
& L_{3}=-i \frac{\partial}{\partial \phi} .
\end{aligned}
$$

These relations can be summarized as

$$
L_{a}=-i \xi_{a}^{\alpha} \partial_{\alpha}
$$

where the Greek index $\alpha$ corresponds to the spherical coordinates and $\xi_{a}^{\alpha}$ are the components of the Killing vector fields associated with the isometries of the sphere. The metric tensor of the sphere can be expressed in terms of the Killing vectors as

$$
g^{\alpha \beta}=\frac{1}{R^{2}} \xi_{a}^{\alpha} \xi_{a}^{\beta}
$$


Any function on the sphere can be expanded in terms of the eigenfunctions of the sphere,

$$
a(\theta, \phi)=\sum_{l=0}^{\infty} \sum_{m=-l}^{l} a_{l m} Y_{l m}(\theta, \phi),
$$

where $a_{l m}$ is a complex coefficient and $Y_{l m}(\theta, \phi)$ are the spherical harmonics, which satisfy the equation

$$
L^{2} Y_{l m}=-R^{2} \Delta_{S^{2}} Y_{l m}=l(l+1) Y_{l m},
$$

where $\Delta_{S^{2}}$ is the scalar Laplacian on the sphere

$$
\Delta_{S^{2}}=\frac{1}{\sqrt{g}} \partial_{a}\left(g^{a b} \sqrt{g} \partial_{b}\right) .
$$

The spherical harmonics have an eigenvalue $\mu \sim l(l+1)$ for integer $l=0,1, \ldots$, with degeneracy $2 l+1$. The orthogonality condition of the spherical harmonics is

$$
\int d \Omega Y_{l m}^{\dagger} Y_{l^{\prime} m^{\prime}}=\delta_{l l^{\prime}} \delta_{m m^{\prime}}
$$

where $d \Omega=\sin \theta d \theta d \phi$.

The spherical harmonics can be expressed in terms of the cartesian coordinates $x_{a}$ of a unit vector in $\mathbb{R}^{3}$,

$$
Y_{l m}(\theta, \phi)=\sum_{\vec{a}} f_{a_{1} \ldots a_{l}}^{(l m)} x^{a_{1}} \cdots x^{a_{l}}
$$

where $f_{a_{1} \ldots a_{l}}^{(l m)}$ is a traceless symmetric tensor of $S O(3)$ with rank $l$.

Similarly we can expand $N \times N$ matrices on a sphere as,

$$
\hat{a}=\sum_{l=0}^{N-1} \sum_{m=-l}^{l} a_{l m} \hat{Y}_{l m}, \quad \hat{Y}_{l m}=R^{-l} \sum_{\vec{a}} f_{a_{1} \ldots a_{l}}^{(l m)} \hat{X}^{a_{1}} \cdots \hat{X}^{a_{l}},
$$

where

$$
\hat{X}_{a}=\frac{2 R}{\sqrt{N^{2}-1}} \lambda_{a}^{(N)}
$$

and $\lambda_{a}^{(N)}$ are the generators of $S U(2)$ in the $N$-dimensional representation. The tensor $f_{\hat{a}_{1} \ldots \hat{a}_{l}}^{(l m)}$ is the same one as in (2.2). The matrices $\hat{Y}_{l m}$ are known as fuzzy spherical harmonics for reasons which will be apparent shortly. They obey the orthonormality condition

$$
\operatorname{Tr}_{N}\left(\hat{Y}_{l m}^{\dagger} \hat{Y}_{l^{\prime} m^{\prime}}\right)=\delta_{l l^{\prime}} \delta_{m m^{\prime}}
$$

There is an obvious relation between equations (2.1) and (2.3), namely ${ }^{1}$

$$
\hat{a}=\sum_{l=0}^{N-1} \sum_{m=-l}^{l} a_{l m} \hat{Y}_{l m} \rightarrow a(\theta, \phi)=\sum_{l=0}^{N-1} \sum_{m=-l}^{l} a_{l m} Y_{l m}(\theta, \phi) .
$$

\footnotetext{
${ }^{1}$ Let us note that in general the map from matrices to functions is not unique, since the expansion coefficients $a_{l m}$ may be different. However, here we introduce the fuzzy sphere by truncating the algebra of functions on the ordinary sphere and therefore the use of the same expansion coefficients is a natural choice.
} 
Notice that the expansion in spherical harmonics is truncated at $N-1$ reflecting the finite number of degrees of freedom in the matrix $\hat{a}$. This allows the consistent definition of a matrix approximation of the sphere known as fuzzy sphere.

According to the above discussion the fuzzy sphere [24] is a matrix approximation of the usual sphere $S^{2}$. The algebra of functions on $S^{2}$ (for example spanned by the spherical harmonics) is truncated at a given frequency and thus becomes finite-dimensional. The truncation has to be consistent with the associativity of the algebra and this can be nicely achieved relaxing the commutativity property of the algebra. The fuzzy sphere is the "space" described by this non-commutative algebra. The algebra itself is that of $N \times N$ matrices, which we denote as $\operatorname{Mat}(N ; \mathbb{C})$. More precisely, the fuzzy sphere $S_{N}^{2}$ at fuzziness level $N-1$ is the non-commutative manifold whose coordinate functions $\hat{X}_{a}$ are $N \times N$ hermitian matrices proportional to the generators of the $N$-dimensional representation of $S U(2)$ as in equation (2.4). They satisfy the condition $\sum_{a=1}^{3} \hat{X}_{a} \hat{X}_{a}=R^{2}$ and the commutation relations

$$
\left[\hat{X}_{a}, \hat{X}_{b}\right]=i \alpha \varepsilon_{a b c} \hat{X}_{c}
$$

where $\alpha=\frac{2 R}{\sqrt{N^{2}-1}}$. It can be proven that for $N \rightarrow \infty$ one obtains the usual commutative sphere. In the following we shall mainly work with the following antihermitian matrices,

$$
X_{a}=\frac{\hat{X}_{a}}{i \alpha R}
$$

which describe equivalently the algebra of the fuzzy sphere and they satisfy the relations

$$
\sum_{a=1}^{3} X_{a} X_{a}=-\frac{1}{\alpha^{2}}, \quad\left[X_{a}, X_{b}\right]=C_{a b c} X_{c}
$$

where $C_{a b c}=\varepsilon_{a b c} / R$.

On the fuzzy sphere there is a natural $S U(2)$ covariant differential calculus. This calculus is three-dimensional and the derivations $e_{a}$ along $X_{a}$ of a function $f$ are given by $e_{a}(f)=\left[X_{a}, f\right]$. Accordingly the action of the Lie derivatives on functions is given by

$$
\mathcal{L}_{a} f=\left[X_{a}, f\right]
$$

these Lie derivatives satisfy the Leibniz rule and the $S U(2)$ Lie algebra relation

$$
\left[\mathcal{L}_{a}, \mathcal{L}_{b}\right]=C_{a b c} \mathcal{L}_{c}
$$

In the $N \rightarrow \infty$ limit the derivations $e_{a}$ become $e_{a}=C_{a b c} x^{b} \partial^{c}$ and only in this commutative limit the tangent space becomes two-dimensional. The exterior derivative is given by

$$
d f=\left[X_{a}, f\right] \theta^{a}
$$

with $\theta^{a}$ the one-forms dual to the vector fields $e_{a},\left\langle e_{a}, \theta^{b}\right\rangle=\delta_{a}^{b}$. The space of one-forms is generated by the $\theta^{a}$ 's in the sense that for any one-form $\omega=\sum_{i} f_{i} d h_{i} t_{i}$ we can always write $\omega=\sum_{a=1}^{3} \omega_{a} \theta^{a}$ with given functions $\omega_{a}$ depending on the functions $f_{i}, h_{i}$ and $t_{i}$. The action of the Lie derivatives $\mathcal{L}_{a}$ on the one-forms $\theta^{b}$ explicitly reads

$$
\mathcal{L}_{a}\left(\theta^{b}\right)=C_{a b c} \theta^{c}
$$

On a general one-form $\omega=\omega_{a} \theta^{a}$ we have $\mathcal{L}_{b} \omega=\mathcal{L}_{b}\left(\omega_{a} \theta^{a}\right)=\left[X_{b}, \omega_{a}\right] \theta^{a}-\omega_{a} C_{b c}^{a} \theta^{c}$ and therefore

$$
\left(\mathcal{L}_{b} \omega\right)_{a}=\left[X_{b}, \omega_{a}\right]-\omega_{c} C_{b a}^{c} .
$$


The differential geometry on the product space Minkowski times fuzzy sphere, $M^{4} \times S_{N}^{2}$, is easily obtained from that on $M^{4}$ and on $S_{N}^{2}$. For example a one-form $A$ defined on $M^{4} \times S_{N}^{2}$ is written as

$$
A=A_{\mu} d x^{\mu}+A_{a} \theta^{a}
$$

with $A_{\mu}=A_{\mu}\left(x^{\mu}, X_{a}\right)$ and $A_{a}=A_{a}\left(x^{\mu}, X_{a}\right)$.

One can also introduce spinors on the fuzzy sphere and study the Lie derivative on these spinors [19]. Although here we have sketched the differential geometry on the fuzzy sphere, one can study other (higher-dimensional) fuzzy spaces (e.g. fuzzy $C P^{M}$ [25], see also [26]) and with similar techniques their differential geometry.

\subsubsection{Gauge theory on the fuzzy sphere}

In order to describe gauge fields on the fuzzy sphere it is natural to introduce the notion of covariant coordinates [27]. In order to do so let us begin with a field $\phi\left(X_{a}\right)$ on the fuzzy sphere, which is a polynomial in the $X_{a}$ coordinates. An infinitesimal gauge transformation $\delta \phi$ of the field $\phi$ with gauge transformation parameter $\lambda\left(X_{a}\right)$ is defined by

$$
\delta \phi(X)=\lambda(X) \phi(X) .
$$

This is an infinitesimal Abelian $U(1)$ gauge transformation if $\lambda(X)$ is just an antihermitian function of the coordinates $X_{a}$, while it is an infinitesimal non-Abelian $U(P)$ gauge transformation if $\lambda(X)$ is valued in $\operatorname{Lie}(U(P))$, the Lie algebra of hermitian $P \times P$ matrices. In the following we will always assume $\operatorname{Lie}(U(P))$ elements to commute with the coordinates $X_{a}$. The coordinates $X_{a}$ are invariant under a gauge transformation

$$
\delta X_{a}=0 .
$$

Then, multiplication of a field on the left by a coordinate is not a covariant operation in the non-commutative case. That is

$$
\delta\left(X_{a} \phi\right)=X_{a} \lambda(X) \phi
$$

and in general the right hand side is not equal to $\lambda(X) X_{a} \phi$. Following the ideas of ordinary gauge theory one then introduces covariant coordinates $\phi_{a}$ such that

$$
\delta\left(\phi_{a} \phi\right)=\lambda \phi_{a} \phi
$$

This happens if

$$
\delta\left(\phi_{a}\right)=\left[\lambda, \phi_{a}\right]
$$

The analogy with ordinary gauge theory also suggests to set

$$
\phi_{a} \equiv X_{a}+A_{a}
$$

and interpret $A_{a}$ as the gauge potential of the non-commutative theory. Then $\phi_{a}$ is the noncommutative analogue of a covariant derivative. The transformation properties of $A_{a}$ support the interpretation of $A_{a}$ as gauge field, since from requirement (2.5) we can deduce that $A_{a}$ transforms as

$$
\delta A_{a}=-\left[X_{a}, \lambda\right]+\left[\lambda, A_{a}\right] .
$$


Correspondingly we can define a tensor $F_{a b}$, the analogue of the field strength, as

$$
F_{a b}=\left[X_{a}, A_{b}\right]-\left[X_{b}, A_{a}\right]+\left[A_{a}, A_{b}\right]-C_{a b}^{c} A_{c}=\left[\phi_{a}, \phi_{b}\right]-C_{a b}^{c} \phi_{c} .
$$

The presence of the last term in (2.6) might seem strange at first sight, however it is imposed in the definition of the field strength by the requirement of covariance. Indeed, it is straightforward to prove that the above tensor transforms covariantly, i.e.

$$
\delta F_{a b}=\left[\lambda, F_{a b}\right] .
$$

Similarly, for a spinor $\psi$ in the adjoint representation, the infinitesimal gauge transformation is given by

$$
\delta \psi=[\lambda, \psi] .
$$

\subsection{Dimensional reduction and gauge symmetry enhancement}

Let us now consider a non-commutative gauge theory on $M^{4} \times(S / R)_{F}$ with gauge group $G=$ $U(P)$ and examine its four-dimensional interpretation. $(S / R)_{F}$ is a fuzzy coset, for example the fuzzy sphere $S_{N}^{2}$. The action is

$$
\mathcal{S}_{\mathrm{YM}}=\frac{1}{4 g^{2}} \int d^{4} x k \operatorname{Tr} \operatorname{tr}_{G} F_{M N} F^{M N}
$$

where $k \operatorname{Tr}$ denotes integration over the fuzzy coset $(S / R)_{F}$ described by $N \times N$ matrices; here the parameter $k$ is related to the size of the fuzzy coset space. For example for the fuzzy sphere we have $R^{2}=\sqrt{N^{2}-1} \pi k$ [9]. In the $N \rightarrow \infty$ limit $k \operatorname{Tr}$ becomes the usual integral on the coset space. For finite $N$, Tr is a good integral because it has the cyclic property $\operatorname{Tr}\left(f_{1} \cdots f_{p-1} f_{p}\right)=\operatorname{Tr}\left(f_{p} f_{1} \cdots f_{p-1}\right)$. It is also invariant under the action of the group $S$, that is infinitesimally given by the Lie derivative. In the action (2.7) $\operatorname{tr}_{G}$ is the gauge group $G$ trace. The higher-dimensional field strength $F_{M N}$, decomposed in four-dimensional space-time and extra-dimensional components, reads as $\left(F_{\mu \nu}, F_{\mu b}, F_{a b}\right)$, where $\mu, \nu$ are four-dimensional spacetime indices. The various components of the field strength are explicitly given by

$$
\begin{aligned}
& F_{\mu \nu}=\partial_{\mu} A_{\nu}-\partial_{\nu} A_{\mu}+\left[A_{\mu}, A_{\nu}\right], \\
& F_{\mu a}=\partial_{\mu} A_{a}-\left[X_{a}, A_{\mu}\right]+\left[A_{\mu}, A_{a}\right], \\
& F_{a b}=\left[X_{a}, A_{b}\right]-\left[X_{b}, A_{a}\right]+\left[A_{a}, A_{b}\right]-C_{a b}^{c} A_{c} .
\end{aligned}
$$

In terms of the covariant coordinates $\phi$, which were introduced in the previous section, the field strength in the non-commutative directions becomes

$$
\begin{aligned}
& F_{\mu a}=\partial_{\mu} \phi_{a}+\left[A_{\mu}, \phi_{a}\right]=D_{\mu} \phi_{a}, \\
& F_{a b}=\left[\phi_{a}, \phi_{b}\right]-C_{a b}^{c} \phi_{c} .
\end{aligned}
$$

Using these expressions the action (2.7) becomes

$$
\mathcal{S}_{\mathrm{YM}}=\int d^{4} x \operatorname{Tr}_{\operatorname{tr}}\left(\frac{k}{4 g^{2}} F_{\mu \nu}^{2}+\frac{k}{2 g^{2}}\left(D_{\mu} \phi_{a}\right)^{2}-V(\phi)\right),
$$

where the potential term $V(\phi)$ is the $F_{a b}$ kinetic term (in our conventions $F_{a b}$ is antihermitian so that $V(\phi)$ is hermitian and non-negative),

$$
V(\phi)=-\frac{k}{4 g^{2}} \operatorname{Tr}_{\operatorname{tr}} \sum_{a b} F_{a b} F_{a b}
$$




$$
=-\frac{k}{4 g^{2}} \operatorname{Tr}_{\operatorname{tr}}\left(\left[\phi_{a}, \phi_{b}\right]\left[\phi^{a}, \phi^{b}\right]-4 C_{a b c} \phi^{a} \phi^{b} \phi^{c}+2 R^{-2} \phi^{2}\right) .
$$

The action (2.8) is naturally interpreted as an action in four dimensions. The infinitesimal $G$ gauge transformation with gauge parameter $\lambda\left(x^{\mu}, X^{a}\right)$ can indeed be interpreted just as an $M^{4}$ gauge transformation. We write

$$
\lambda\left(x^{\mu}, X^{a}\right)=\lambda^{I}\left(x^{\mu}, X^{a}\right) \mathcal{T}^{I}=\lambda^{h, I}\left(x^{\mu}\right) T^{h} \mathcal{T}^{I},
$$

where $\mathcal{T}^{I}$ are hermitian generators of $U(P), \lambda^{I}\left(x^{\mu}, X^{a}\right)$ are $N \times N$ antihermitian matrices and thus are expressible as $\lambda\left(x^{\mu}\right)^{I, h} T^{h}$, where $T^{h}$ are antihermitian generators of $U(N)$. The fields $\lambda\left(x^{\mu}\right)^{I, h}$, with $h=1, \ldots, N^{2}$, are the Kaluza-Klein modes of $\lambda\left(x^{\mu}, X^{a}\right)^{I}$. We now consider on equal footing the indices $h$ and $I$ and interpret the fields on the r.h.s. of (2.10) as one field valued in the tensor product Lie algebra $\operatorname{Lie}(U(N)) \otimes \operatorname{Lie}(U(P))$. This Lie algebra is indeed $\operatorname{Lie}(U(N P))$ (the $(N P)^{2}$ generators $T^{h} \mathcal{T}^{I}$ being $N P \times N P$ antihermitian matrices that are linear independent). Similarly we rewrite the gauge field $A_{\nu}$ as

$$
A_{\nu}\left(x^{\mu}, X^{a}\right)=A_{\nu}^{I}\left(x^{\mu}, X^{a}\right) \mathcal{T}^{I}=A_{\nu}^{h, I}\left(x^{\mu}\right) T^{h} \mathcal{T}^{I},
$$

and interpret it as a $\operatorname{Lie}(U(N P))$-valued gauge field on $M^{4}$. The four-dimensional scalar fields $\phi_{a}$ are interpreted similarly. It is worth noting that the scalars transform in the adjoint representation of the four-dimensional gauge group and therefore they are not suitable for the electroweak symmetry breaking. This serves as a motivation to use a non-trivial dimensional reduction scheme, which is presented in the following section. Finally $\operatorname{Tr}_{G} \operatorname{tr}_{G}$ is the trace over $U(N P)$ matrices in the fundamental representation.

Up to now we have just performed a ordinary fuzzy dimensional reduction. Indeed in the commutative case the expression (2.8) corresponds to rewriting the initial lagrangian on $M^{4} \times S^{2}$ using spherical harmonics on $S^{2}$. Here the space of functions is finite-dimensional and therefore the infinite tower of modes reduces to the finite sum given by the trace Tr. The remarkable result of the above analysis is that the gauge group in four dimensions is enhanced compared to the gauge group $G$ in the higher-dimensional theory. Therefore it is very interesting to note that we can in fact start with an Abelian gauge group in higher dimensions and obtain non-Abelian gauge symmetry in the four-dimensional theory.

\subsection{Non-trivial dimensional reduction over fuzzy extra dimensions}

In this section we reduce the number of gauge fields and scalars in the action (2.8) by applying the Coset Space Dimensional Reduction (CSDR) scheme. Before proceeding to the case of fuzzy extra dimensions let us briefly recall how this scheme works in the commutative case.

\subsubsection{Ordinary CSDR}

One way to dimensionally reduce a gauge theory on $M^{4} \times S / R$ with gauge group $G$ to a gauge theory on $M^{4}$, is to consider field configurations that are invariant under $S / R$ transformations. Since the action of the group $S$ on the coset space $S / R$ is transitive (i.e., connects all points), we can equivalently require the fields in the theory to be invariant under the action of $S$ on $S / R$. Infinitesimally, if we denote by $\xi_{a}$ the Killing vectors on $S / R$ associated to the generators $T^{a}$ of $S$, we require the fields to have zero Lie derivative along $\xi_{a}$. For scalar fields this is equivalent to requiring independence under the $S / R$ coordinates. The CSDR scheme dimensionally reduces a gauge theory on $M^{4} \times S / R$ with gauge group $G$ to a gauge theory on $M^{4}$ imposing a milder constraint, namely the fields are required to be invariant under the $S$ action up to a $G$ gauge 
transformation $[3,4,5]^{2}$. Thus we have, respectively for scalar fields $\phi$ and the one-form gauge field $A$,

$$
\mathcal{L}_{\xi_{a}} \phi=\delta^{W_{a}} \phi=W_{a} \phi, \quad \mathcal{L}_{\xi_{a}} A=\delta^{W_{a}} A=-D W_{a},
$$

where $\delta^{W_{a}}$ is the infinitesimal gauge transformation relative to the gauge parameter $W_{a}$ that depends on the coset coordinates (in our notations $A$ and $W_{a}$ are antihermitian and the covariant derivative reads $D=d+A$ ). The gauge parameters $W_{a}$ obey a consistency condition which follows from the relation

$$
\left[\mathcal{L}_{\xi_{a}}, \mathcal{L}_{\xi_{b}}\right]=\mathcal{L}_{\left[\xi_{a}, \xi_{b}\right]}
$$

and transform under a gauge transformation $\phi \rightarrow g \phi$ as

$$
W_{a} \rightarrow g W_{a} g^{-1}+\left(\mathcal{L}_{\xi_{a}} g\right) g^{-1} .
$$

Since two points of the coset are connected by an $S$-transformation which is equivalent to a gauge transformation, and since the Lagrangian is gauge invariant, we can study the above equations just at one point of the coset, let's say $y^{\alpha}=0$, where we denote by $\left(x^{\mu}, y^{\alpha}\right)$ the coordinates of $M^{4} \times S / R$, and we use $a, \alpha, i$ to denote $S, S / R$ and $R$ indices. In general, using (2.13), not all the $W_{a}$ can be gauged transformed to zero at $y^{\alpha}=0$, however one can choose $W_{\alpha}=0$ denoting by $W_{i}$ the remaining ones. Then the consistency condition which follows from equation (2.12) implies that $W_{i}$ are constant and equal to the generators of the embedding of $R$ in $G$ (thus in particular $R$ must be embeddable in $G$; we write $R_{G}$ for the image of $R$ in $G$ ).

The detailed analysis of the constraints given in $[3,4]$ provides us with the four-dimensional unconstrained fields as well as with the gauge invariance that remains in the theory after dimensional reduction. Here we just state the results:

- The components $A_{\mu}(x, y)$ of the initial gauge field $A_{M}(x, y)$ become, after dimensional reduction, the four-dimensional gauge fields and furthermore they are independent of $y$. In addition one can find that they have to commute with the elements of the $R_{G}$ subgroup of $G$. Thus the four-dimensional gauge group $H$ is the centralizer of $R$ in $G, H=C_{G}\left(R_{G}\right)$.

- Similarly, the $A_{\alpha}(x, y)$ components of $A_{M}(x, y)$ denoted by $\phi_{\alpha}(x, y)$ from now on, become scalars in four dimensions. These fields transform under $R$ as a vector $v$, i.e.

$$
\begin{aligned}
& S \supset R, \\
& \operatorname{adj} S=\operatorname{adj} R+v .
\end{aligned}
$$

Moreover $\phi_{\alpha}(x, y)$ acts as an intertwining operator connecting induced representations of $R$ acting on $G$ and $S / R$. This implies, exploiting Schur's lemma, that the transformation properties of the fields $\phi_{\alpha}(x, y)$ under $H$ can be found if we express the adjoint representation of $G$ in terms of $R_{G} \times H$ :

$$
\begin{aligned}
& G \supset R_{G} \times H, \\
& \operatorname{adj} G=(\operatorname{adj} R, 1)+(1, \operatorname{adj} H)+\sum\left(r_{i}, h_{i}\right) .
\end{aligned}
$$

Then if $v=\sum s_{i}$, where each $s_{i}$ is an irreducible representation of $R$, there survives an $h_{i}$ multiplet for every pair $\left(r_{i}, s_{i}\right)$, where $r_{i}$ and $s_{i}$ are identical irreps. of $R$. If we start from a pure gauge theory on $M^{4} \times S / R$, the four-dimensional potential (at $y^{\alpha}=0$ ) can be shown to be given by

$$
V=-\frac{1}{4} F_{\alpha \beta} F^{\alpha \beta}=-\frac{1}{4}\left(C^{c}{ }_{\alpha \beta} \phi_{c}-\left[\phi_{\alpha}, \phi_{\beta}\right]\right)^{2},
$$

\footnotetext{
${ }^{2}$ See also [28] for related work.
} 
where we have defined $\phi_{i} \equiv W_{i}$. However, the fields $\phi_{\alpha}$ are not independent because the conditions $(2.11)$ at $y^{\alpha}=0$ constrain them. The solution of the constraints provides the physical dimensionally reduced fields in four dimensions; in terms of these physical fields the potential is still a quartic polynomial. Then, the minimum of this potential will determine the spontaneous symmetry breaking pattern.

- Turning next to the fermion fields, similarly to scalars, they act as an intertwining operator connecting induced representations of $R$ in $G$ and in $S O(d)$, where $d$ is the dimension of the tangent space of $S / R$. Proceeding along similar lines as in the case of scalars, and considering the more interesting case of even dimensions, we impose first the Weyl condition. Then to obtain the representation of $H$ under which the four-dimensional fermions transform, we have to decompose the fermion representation $F$ of the initial gauge group $G$ under $R_{G} \times H$, i.e.

$$
F=\sum\left(t_{i}, h_{i}\right)
$$

and the spinor of $S O(d)$ under $R$

$$
\sigma_{d}=\sum \sigma_{j}
$$

Then for each pair $t_{i}$ and $\sigma_{i}$, where $t_{i}$ and $\sigma_{i}$ are identical irreps. there is an $h_{i}$ multiplet of spinor fields in the four-dimensional theory. In order however to obtain chiral fermions in the effective theory we have to impose further requirements $[4,7]$. The issue of chiral fermions will be discussed in Section 2.4.

\subsubsection{Fuzzy CSDR}

Let us now discuss how the above scheme can be applied in the case where the extra dimensions are fuzzy coset spaces $[19]^{3}$. Since $S U(2)$ acts on the fuzzy sphere $(S U(2) / U(1))_{F}$, and more in general the group $S$ acts on the fuzzy coset $(S / R)_{F}$, we can state the CSDR principle in the same way as in the continuum case, i.e. the fields in the theory must be invariant under the infinitesimal $S U(2)$, respectively $S$, action up to an infinitesimal gauge transformation

$$
\begin{aligned}
& \mathcal{L}_{b} \phi=\delta_{W_{b}} \phi=W_{b} \phi, \\
& \mathcal{L}_{b} A=\delta_{W_{b}} A=-D W_{b},
\end{aligned}
$$

where $A$ is the one-form gauge potential $A=A_{\mu} d x^{\mu}+A_{a} \theta^{a}$, and $W_{b}$ depends only on the coset coordinates $X^{a}$ and (like $A_{\mu}, A_{a}$ ) is antihermitian. We thus write $W_{b}=W_{b}^{I} \mathcal{T}^{I}, I=1,2, \ldots, P^{2}$, where $\mathcal{T}^{I}$ are hermitian generators of $U(P)$ and $\left(W_{b}^{I}\right)^{\dagger}=-W_{b}^{I}$; here ${ }^{\dagger}$ is hermitian conjugation on the $X^{a}$ 's.

In terms of the covariant coordinate $\phi_{a}=X_{a}+A_{a}$ and of

$$
\omega_{a} \equiv X_{a}-W_{a},
$$

the CSDR constraints (2.14) and (2.15) assume a particularly simple form, namely

$$
\begin{aligned}
& {\left[\omega_{b}, A_{\mu}\right]=0,} \\
& C_{b d e} \phi^{e}=\left[\omega_{b}, \phi_{d}\right] .
\end{aligned}
$$

In addition we have a consistency condition following from the relation $\left[\mathcal{L}_{a}, \mathcal{L}_{b}\right]=C_{a b}{ }^{c} \mathcal{L}_{c}$ :

$$
\left[\omega_{a}, \omega_{b}\right]=C_{a b}^{c} \omega_{c},
$$

where $\omega_{a}$ transforms as $\omega_{a} \rightarrow \omega_{a}^{\prime}=g \omega_{a} g^{-1}$. One proceeds in a similar way for the spinor fields [19].

\footnotetext{
${ }^{3} \mathrm{~A}$ similar approach has also been considered in [29].
} 


\subsubsection{Solving the CSDR constraints for the fuzzy sphere}

We consider $(S / R)_{F}=S_{N}^{2}$, i.e. the fuzzy sphere, and to be definite at fuzziness level $N-1(N \times N$ matrices). We study here the basic example where the gauge group is $G=U(1)$. In this case the $\omega_{a}=\omega_{a}\left(X^{b}\right)$ appearing in the consistency condition (2.18) are $N \times N$ antihermitian matrices and therefore can be interpreted as elements of $\operatorname{Lie}(U(N))$. On the other hand the $\omega_{a}$ satisfy the commutation relations (2.18) of $\operatorname{Lie}(S U(2))$. Therefore in order to satisfy the consistency condition (2.18) we have to embed $\operatorname{Lie}(S U(2))$ in $\operatorname{Lie}(U(N))$. Let $T^{h}$ with $h=1, \ldots,(N)^{2}$ be the generators of $\operatorname{Lie}(U(N))$ in the fundamental representation. Then we can always use the convention $h=(a, u)$ with $a=1,2,3$ and $u=4,5, \ldots, N^{2}$ where the $T^{a}$ satisfy the $S U(2)$ Lie algebra,

$$
\left[T^{a}, T^{b}\right]=C^{a b} T^{c} .
$$

Then we define an embedding by identifying

$$
\omega_{a}=T_{a} .
$$

The constraint (2.16), $\left[\omega_{b}, A_{\mu}\right]=0$, then implies that the four-dimensional gauge group $K$ is the centralizer of the image of $S U(2)$ in $U(N)$, i.e.

$$
K=C_{U(N)}(S U((2)))=S U(N-2) \times U(1) \times U(1),
$$

where the last $U(1)$ is the $U(1)$ of $U(N) \simeq S U(N) \times U(1)$. The functions $A_{\mu}(x, X)$ are arbitrary functions of $x$ but the $X$ dependence is such that $A_{\mu}(x, X)$ is $\operatorname{Lie}(K)$-valued instead of $\operatorname{Lie}(U(N))$, i.e. eventually we have a four-dimensional gauge potential $A_{\mu}(x)$ with values in Lie $(K)$. Concerning the constraint (2.17), it is satisfied by choosing

$$
\phi_{a}=r \phi(x) \omega_{a},
$$

i.e. the unconstrained degrees of freedom correspond to the scalar field $\phi(x)$ which is a singlet under the four-dimensional gauge group $K$.

The choice (2.20) defines one of the possible embedding of $\operatorname{Lie}(S U(2))$ in $\operatorname{Lie}(U(N))$ ). For example, we could also embed $\operatorname{Lie}(S U(2))$ in $\operatorname{Lie}(U(N))$ using the irreducible $N$-dimensional rep. of $S U(2)$, i.e. we could identify $\omega_{a}=X_{a}$. The constraint (2.16) in this case implies that the four-dimensional gauge group is $U(1)$ so that $A_{\mu}(x)$ is $U(1)$ valued. The constraint (2.17) leads again to the scalar singlet $\phi(x)$.

In general, we start with a $U(1)$ gauge theory on $M^{4} \times S_{N}^{2}$. We solve the CSDR constraint (2.18) by embedding $S U(2)$ in $U(N)$. There exist $p_{N}$ embeddings, where $p_{N}$ is the number of ways one can partition the integer $N$ into a set of non-increasing positive integers [24]. Then the constraint (2.16) gives the surviving four-dimensional gauge group. The constraint (2.17) gives the surviving four-dimensional scalars and equation (2.21) is always a solution but in general not the only one. By setting $\phi_{a}=\omega_{a}$ we obtain always a minimum of the potential. This minimum is given by the chosen embedding of $S U(2)$ in $U(N)$.

Concerning fermions in the adjoint, the corresponding analysis in [19] shows that we have to consider the embedding

$$
S \subset S O(\operatorname{dim} S)
$$

which is given by $T_{a}=\frac{1}{2} C_{a b c} \Gamma^{b c}$ that satisfies the commutation relation (2.19). Therefore $\psi$ is an intertwining operator between induced representations of $S$ in $U(N P)$ and in $S O(\operatorname{dim} S)$. To find the surviving fermions, as in the commutative case [4], we decompose the adjoint rep. of $U(N P)$ under $S_{U(N P)} \times K$,

$$
U(N P) \supset S_{U(N P)} \times K,
$$




$$
\operatorname{adj} U(N P)=\sum_{i}\left(s_{i}, k_{i}\right)
$$

We also decompose the spinor rep. $\sigma$ of $S O(\operatorname{dim} S)$ under $S$

$$
\begin{aligned}
& S O(\operatorname{dim} S) \supset S, \\
& \sigma=\sum_{e} \sigma_{e} .
\end{aligned}
$$

Then, when we have two identical irreps. $s_{i}=\sigma_{e}$, there is a $k_{i}$ multiplet of fermions surviving in four dimensions, i.e. four-dimensional spinors $\psi(x)$ belonging to the $k_{i}$ representation of $K$.

An important point that we would like to stress here is the question of the renormalizability of the gauge theory defined on $M_{4} \times(S / R)_{F}$. First we notice that the theory exhibits certain features so similar to a higher-dimensional gauge theory defined on $M_{4} \times S / R$ that naturally it could be considered as a higher-dimensional theory too. For instance the isometries of the spaces $M_{4} \times S / R$ and $M_{4} \times(S / R)_{F}$ are the same. It does not matter if the compact space is fuzzy or not. For example in the case of the fuzzy sphere, i.e. $M_{4} \times S_{N}^{2}$, the isometries are $S O(3,1) \times S O(3)$ as in the case of the continuous space, $M_{4} \times S^{2}$. Similarly the coupling of a gauge theory defined on $M_{4} \times S / R$ and on $M_{4} \times(S / R)_{F}$ are both dimensionful and have exactly the same dimensionality. On the other hand the first theory is clearly non-renormalizable, while the latter is renormalizable (in the sense that divergencies can be removed by a finite number of counterterms). So from this point of view one finds a partial justification of the old hopes for considering quantum field theories on non-commutative structures. If this observation can lead to finite theories too, it remains as an open question.

\subsection{The problem of chirality in fuzzy CSDR}

Among the great successes of the ordinary CSDR is the possibility to accommodate chiral fermions in the four-dimensional theory [7]. Needless to say that the requirement of chirality for the four-dimensional fermions is necessary in order for a theory to have a chance to become realistic.

Let us recall the necessary conditions for accommodating chiral fermions in four dimensions when a higher-dimensional gauge theory with gauge group $G$ is reduced over a $d$-dimensional coset space $S / R$ using the CSDR scheme. As we discussed previously, solving the CSDR constraints for the fermion fields leads to the result that in order to obtain the representations of the four-dimensional unbroken gauge group $H$ under which the four-dimensional fermions transform, we have to decompose the representation $F$ of the initial gauge group in which the fermions are assigned under $R \times H$, i.e.

$$
F=\sum\left(t_{i}, h_{i}\right)
$$

and the spinor of the tangent space group $S O(d)$ under $R$

$$
\sigma_{d}=\sum \sigma_{j}
$$

Then for each pair $t_{i}$ and $\sigma_{i}$, where $t_{i}$ and $\sigma_{i}$ are identical irreducible representations there is an $h_{i}$ multiplet of spinor fields in the four-dimensional theory.

In order to obtain chiral fermions in four dimensions we need some further requirements. The representation of interest, for our purposes, of the spin group is the spinor representation. This

has dimensions $2^{\frac{d}{2}}$ and $2^{\frac{(d-1)}{2}}$ for $d$ even and odd respectively. For odd $d$ the representation is irreducible but for even $d$ it is reducible into two irreducible components of equal dimension. This splitting exactly gives the possibility to define Weyl spinors and to construct a chirality 
operator. Thus if we are in odd number of dimensions (where the chirality operator does not exist) there is no way to obtain chiral fermions. For this reason we focus only on even dimensions.

The first possibility is to start with Dirac fermions in $D$ (even) dimensions. Here we can define the standard chirality operator

$$
\Gamma^{D+1}=i^{\frac{D(D-1)}{2}} \Gamma^{1} \Gamma^{2} \cdots \Gamma^{D},
$$

with $\left(\Gamma^{D+1}\right)^{2}=1$ and $\left\{\Gamma^{D+1}, \Gamma^{A}\right\}=0$, where $\Gamma^{A}, A=1, \ldots, D$ span the Clifford algebra in $D$ dimensions. This operator has eigenvalues \pm 1 and distinguishes left and right spinors. So, it is possible to define a Weyl basis, where the chirality operator is diagonal, namely

$$
\Gamma^{D+1} \psi_{ \pm}= \pm \psi_{ \pm}
$$

As we mentioned above, in this case $S O(1, D-1)$ has two independent irreducible spinor representations, $\sigma_{D}$ and $\sigma_{D}^{\prime}$, under which the Weyl spinors $\psi_{+}$and $\psi_{-}$transform respectively. The following branching rule for the spinors holds ${ }^{4}$

$$
\begin{aligned}
& S O(1, D-1) \supset S O(1,3) \times S O(d), \\
& \sigma_{D}=\left(2,1 ; \sigma_{d}\right)+\left(1,2 ; \sigma_{d}^{\prime}\right), \\
& \sigma_{D}^{\prime}=\left(2,1 ; \sigma_{d}^{\prime}\right)+\left(1,2 ; \sigma_{d}\right) .
\end{aligned}
$$

Then, since we started with a Dirac spinor $\psi=\psi_{+} \oplus \psi_{-}$transforming under a representation $F$ of the original gauge group $G$, following the rule which was stated above it is obvious that we obtain fermions in four dimensions appearing in equal numbers of left and right representations of the unbroken gauge group $H$. Thus, starting with Dirac fermions does not render the fermions of the four-dimensional theory chiral.

In order to overcome this problem we can make a further restriction and start with Weyl fermions, namely to impose the Weyl condition in higher dimensions. Then, only one of the $\sigma_{D}$ and $\sigma_{D}^{\prime}$ representations is selected. There are still two cases to investigate, the total number of dimensions being $4 n$ or $4 n+2$. Since we are interested in vacuum configurations of the form $M^{4} \times S / R$ the dimensionality of the internal (coset) space is then of the form $4 n$ or $4 n+2$ respectively.

For $D=4 n(d=4(n-1))$, the two spinor representations of $S O(d)$ are self-conjugate, meaning that in the decomposition

$$
\begin{aligned}
& S O(d) \supset R, \\
& \sigma_{d}=\sum \sigma_{i},
\end{aligned}
$$

$\sigma_{i}$ is either a real representation or it appears together with its conjugate representation $\bar{\sigma}_{i}$. Thus we are led to consider that the representation $F$ of $G$ where the fermions are assigned has to be complex. Two important things to note is that $R$ is also required to admit complex representations (otherwise the decompositions of $\sigma_{d}$ and $\sigma_{d}^{\prime}$ will be the same, leading to a nonchiral theory) and that $\operatorname{rank} S=\operatorname{rank} R$ (otherwise $\sigma_{d}$ and $\sigma_{d}^{\prime}$ will again be the same). These requirements still hold in the following case.

In the case $D=4 n+2(d=4(n-1)+2)$, the two spinor representations of $S O(d)$ are not self-conjugate anymore and $\sigma_{d}^{\prime}=\bar{\sigma}_{d}$. Now, the decomposition reads as

$$
S O(d) \supset R
$$

\footnotetext{
${ }^{4}$ Here the usual notation for two-component Weyl spinors of the Lorentz group $S O(1,3)$ is adopted, namely $\psi_{+} \rightarrow(1,2)$ and $\psi_{-} \rightarrow(2,1)$.
} 


$$
\begin{aligned}
\sigma_{d} & =\sum \sigma_{i}, \\
\bar{\sigma}_{d} & =\sum \bar{\sigma}_{i},
\end{aligned}
$$

so we can let $F$ be a vectorlike representation. Then, in the decomposition

$$
\begin{aligned}
& G \supset R_{G} \times H, \\
& F=\sum\left(t_{i}, h_{i}\right),
\end{aligned}
$$

each term $\left(t_{i}, h_{i}\right)$ will either be self-conjugate or it will appear with the term $\left(\bar{t}_{i}, \bar{h}_{i}\right)$. According to the established rule, $\sigma_{d}$ will provide a left-handed fermion multiplet transforming under the four-dimensional gauge group as $f_{L}=\sum h_{i}^{L}$; $\bar{\sigma}_{d}$ will provide a right-handed fermion multiplet transforming as $f_{R}=\sum \bar{h}_{i}^{R}$. Since $h_{i}^{L} \sim \bar{h}_{i}^{R}$ we are led to two Weyl fermions with the same chirality in the same representation of the unbroken gauge group $H$. This is of course a chiral theory, which is the desired result. Moreover, the doubling of the fermions can be eliminated by imposing the Majorana condition, if applicable ${ }^{5}$.

Let us use the same spirit in order to investigate the possibility of obtaining chiral fermions in the fuzzy case as well. We discussed previously that we have to consider the embedding

$$
S \subset S O(\operatorname{dim} S)
$$

concerning fermions in the adjoint. In order to determine the surviving fermions, as in the commutative case, we decompose the adjoint rep. of $U(N)$ under $S_{U(N)} \times K$,

$$
\begin{aligned}
& U(N) \supset S_{U(N)} \times K, \\
& \operatorname{adj} U(N)=\sum_{i}\left(s_{i}, k_{i}\right) .
\end{aligned}
$$

We also decompose the spinor rep. $\sigma$ of $S O(\operatorname{dim} S)$ under $S$

$$
\begin{aligned}
& S O(\operatorname{dim} S) \supset S, \\
& \sigma=\sum_{e} \sigma_{e} .
\end{aligned}
$$

Then, when we have two identical irreps. $s_{i}=\sigma_{e}$, there is a $k_{i}$ multiplet of fermions surviving in four dimensions, i.e. four-dimensional spinors $\psi(x)$ belonging in the $k_{i}$ representation of $K$.

Concerning the issue of chirality, the situation is now different. The main difference is obviously the modification of the rule for the surviving fermions. In the continuous case we had to embed $R$ in $S O(d)$, while now the suitable embedding is that of $S$ in $S O(\operatorname{dim} S)$. Exploring chirality in the continuous case, we had to deal with the representations of $S O(d)$. Recall that we required $d$ to be even so that there are two independent spinor representations; therefore in the fuzzy case we require $\operatorname{dim} S$ to be even. Moreover, when $d=4 n$ we concluded that the representation $F$, where the fermions are initially assigned, has to be complex. Since in the fuzzy case we assign the fermions in the adjoint representation, the case $\operatorname{dim} S=4 n$ would lead to a non-chiral theory. Finally, the case $\operatorname{dim} S=4 n+2$ is the only promising one and one would expect to obtain chiral fermions, as in the continuous case when $d=4 n+2$. However, we also need the further requirement that $S$ admits complex representations, again in analogy with $R$ admitting complex representations in the continuous case.

In summary, in order to have a chance to obtain chiral fermions in the case of fuzzy extra dimensions the necessary requirements are:

\footnotetext{
${ }^{5}$ Let us remind that the Majorana condition can be imposed when the number of dimensions is $D=2,3,8 n+4$.
} 
- $\operatorname{dim} S=4 n+2$,

- $S$ admits complex irreps.

The above requirements are quite restrictive; for example they are not satisfied in the case of a single fuzzy sphere. In general, using elementary number theory one can show that they cannot be satisfied for any $S$ being a $S U(n), S O(n)$ or $S p(n)$ group. Therefore only products of fuzzy spaces have a chance to lead to chiral fermions after dimensional reduction without further requirements. The simplest case which satisfies these requirements is that of a product of two fuzzy spheres, which will be discussed in Section 3.4 in the context of dynamical generation of fuzzy extra dimensions.

In conclusion it is worth making the following remark. As we saw above, a major difference between fuzzy and ordinary CSDR is that in the fuzzy case one always embeds $S$ in the gauge group $G$ instead of embedding just $R$ in $G$. A generic feature of the ordinary CSDR in the special case when $S$ is embedded in $G$ is that the fermions in the final theory are massive [30]. According to the discussion in Section 2.3.3 the situation in the fuzzy case is very similar to the one we just described. In fuzzy CSDR the spontaneous symmetry breaking mechanism takes already place by solving the fuzzy CSDR constraints. Therefore in the Yukawa sector of the theory we have the results of the spontaneous symmetry breaking, i.e. massive fermions and Yukawa interactions among fermions and the physical Higgs field. We shall revisit the problem of chirality in the following section and finally, in Section 4, we shall describe a way to overcome it and obtain chiral four-dimensional theories.

\section{Dynamical generation of fuzzy extra dimensions}

Let us now discuss a further development [20] of these ideas, which addresses in detail the questions of quantization and renormalization. This leads to a slightly modified model with an extra term in the potential, which dynamically selects a unique (nontrivial) vacuum out of the many possible CSDR solutions, and moreover generates a magnetic flux on the fuzzy sphere. It also allows to show that the full tower of Kaluza-Klein modes is generated on $S_{N}^{2}$. Moreover, upon including fermions, the model offers the possibility of a detailed study of the fermionic sector [21]. Such a study reveals the difficulty in obtaining chiral low-energy models but at the same time it paves the way out of this problem. Indeed, we shall see in the following section that using orbifold techniques it is possible to construct chiral models in the framework of dynamically generated fuzzy extra dimensions.

\subsection{The four dimensional action}

We start with a $S U(N)$ gauge theory on four dimensional Minkowski space $M^{4}$ with coordinates $y^{\mu}, \mu=0,1,2,3$. The action under consideration is

$$
\mathcal{S}_{Y M}=\int d^{4} y \operatorname{Tr}\left(\frac{1}{4 g^{2}} F_{\mu \nu}^{\dagger} F_{\mu \nu}+\left(D_{\mu} \phi_{a}\right)^{\dagger} D_{\mu} \phi_{a}\right)-V(\phi),
$$

where $A_{\mu}$ are $S U(N)$-valued gauge fields, $D_{\mu}=\partial_{\mu}+\left[A_{\mu}, \cdot\right]$, and

$$
\phi_{a}=-\phi_{a}^{\dagger}, \quad a=1,2,3
$$

are three antihermitian scalars in the adjoint of $S U(N)$,

$$
\phi_{a} \rightarrow U^{\dagger} \phi_{a} U,
$$


where $U=U(y) \in S U(N)$. Furthermore, the $\phi_{a}$ transform as vectors of an additional global $S O(3)$ symmetry. The potential $V(\phi)$ is taken to be the most general renormalizable action invariant under the above symmetries, which is

$$
\begin{aligned}
V(\phi)= & \operatorname{Tr}\left(g_{1} \phi_{a} \phi_{a} \phi_{b} \phi_{b}+g_{2} \phi_{a} \phi_{b} \phi_{a} \phi_{b}-g_{3} \varepsilon_{a b c} \phi_{a} \phi_{b} \phi_{c}+g_{4} \phi_{a} \phi_{a}\right) \\
& +\frac{g_{5}}{N} \operatorname{Tr}\left(\phi_{a} \phi_{a}\right) \operatorname{Tr}\left(\phi_{b} \phi_{b}\right)+\frac{g_{6}}{N} \operatorname{Tr}\left(\phi_{a} \phi_{b}\right) \operatorname{Tr}\left(\phi_{a} \phi_{b}\right)+g_{7} .
\end{aligned}
$$

This may not look very transparent at first sight, however it can be written in a very intuitive way. First, we make the scalars dimensionless by rescaling

$$
\phi_{a}^{\prime}=R \phi_{a},
$$

where $R$ has dimension of length; we will usually suppress $R$ since it can immediately be reinserted, and drop the prime from now on. Now observe that for a suitable choice of $R$,

$$
R=\frac{2 g_{2}}{g_{3}},
$$

the potential can be rewritten as

$$
V(\phi)=\operatorname{Tr}\left(a^{2}\left(\phi_{a} \phi_{a}+\tilde{b} \mathbb{1}\right)^{2}+c+\frac{1}{\tilde{g}^{2}} F_{a b}^{\dagger} F_{a b}\right)+\frac{h}{N} g_{a b} g_{a b}
$$

for suitable constants $a, b, c, \tilde{g}, h$, where

$$
F_{a b}=\left[\phi_{a}, \phi_{b}\right]-\varepsilon_{a b c} \phi_{c}=\varepsilon_{a b c} F_{c}, \quad \tilde{b}=b+\frac{d}{N} \operatorname{Tr}\left(\phi_{a} \phi_{a}\right), \quad g_{a b}=\operatorname{Tr}\left(\phi_{a} \phi_{b}\right) .
$$

We will omit $c$ from now. Notice that two couplings were reabsorbed in the definitions of $R$ and $\tilde{b}$. The potential is clearly positive definite provided

$$
a^{2}=g_{1}+g_{2}>0, \quad \frac{2}{\tilde{g}^{2}}=-g_{2}>0, \quad h \geq 0,
$$

which we assume from now on. Here $\tilde{b}=\tilde{b}(y)$ is a scalar, $g_{a b}=g_{a b}(y)$ is a symmetric tensor under the global $S O(3)$, and $F_{a b}=F_{a b}(y)$ is a $s u(N)$-valued antisymmetric tensor field which will be interpreted as field strength in some dynamically generated extra dimensions below. In this form, $V(\phi)$ looks like the action of Yang-Mills gauge theory on a fuzzy sphere in the matrix formulation $[31,32,33,34]$. It differs from the potential in (2.9) only by the presence of the first term $a^{2}\left(\phi_{a} \phi_{a}+\tilde{b}\right)^{2}$, which is strongly suggested by renormalization. In fact it is necessary for the interpretation as pure YM action, and we will see that it is very welcome on physical grounds since it dynamically determines and stabilizes a vacuum, which can be interpreted as extra-dimensional fuzzy sphere. In particular, it removes unwanted flat directions.

\subsection{Emergence of extra dimensions and the fuzzy sphere}

The vacuum of the above model is given by the minimum of the potential (3.1). Finding the minimum of the potential is a rather nontrivial task, and the answer depends crucially on the parameters in the potential [20]. The conditions for the global minimum imply that $\phi_{a}$ is a representation of $S U(2)$, with Casimir $\tilde{b}$ (where it was assumed for simplicity $h=0$ ). Then, it is easy to write down a large class of solutions to the minimum of the potential, by noting that any decomposition of $N=n_{1} N_{1}+\cdots+n_{h} N_{h}$ into irreps of $S U(2)$ with multiplicities $n_{i}$ leads to a block-diagonal solution

$$
\phi_{a}=\operatorname{diag}\left(\alpha_{1} X_{a}^{\left(N_{1}\right)}, \ldots, \alpha_{k} X_{a}^{\left(N_{k}\right)}\right)
$$

of the vacuum equations, where $\alpha_{i}$ are suitable constants which will be determined below.

It turns out [20] that there are essentially only 2 types of vacua: 
1. Type I vacuum: It is plausible that the solution (3.2) with minimal potential contains only representations whose Casimirs are close to $\tilde{b}$. In particular, let $M$ be the dimension of the irrep whose Casimir $C_{2}(M) \approx \tilde{b}$ is closest to $\tilde{b}$. If furthermore the dimensions match as $N=M n$, we expect that the vacuum is given by $n$ copies of the irrep $(M)$, which can be written as $\phi_{a}=\alpha X_{a}^{(M)} \otimes \mathbb{1}_{n}$ with low-energy gauge group $S U(n)$.

2. Type II vacuum: Consider again a solution (3.2) with $n_{i}$ blocks of size $N_{i}=\tilde{N}+m_{i}$, where $\tilde{N}$ is defined by $\tilde{b}=\frac{1}{4}\left(\tilde{N}^{2}-1\right)$, and assume that $\tilde{N}$ is large and $\frac{m_{i}}{\tilde{N}} \ll 1$. The action is then given by

$$
V(\phi)=\operatorname{Tr}\left(\frac{1}{2 \tilde{g}^{2}} \sum_{i} n_{i} m_{i}^{2} \mathbb{1}_{N_{i}}+O\left(\frac{1}{N_{i}}\right)\right) \approx \frac{1}{2 \tilde{g}^{2}} \frac{N}{k} \sum_{i} n_{i} m_{i}^{2},
$$

where $k=\sum n_{i}$ is the total number of irreps, and the solution can be interpreted in terms of "instantons" (non-Abelian monopoles) on the internal fuzzy sphere [31]. Hence in order to determine the solution of type (3.2) with minimal action, we simply have to minimize $\sum_{i} n_{i} m_{i}^{2}$, where the $m_{i} \in \mathbb{Z}-\tilde{N}$ satisfy the constraint $\sum n_{i} m_{i}=N-k \tilde{N}$. In this case the solution with minimal potential among all possible partitions (3.2) is given by

$$
\phi_{a}=\left(\begin{array}{cc}
\alpha_{1} X_{a}^{\left(N_{1}\right)} \otimes \mathbb{1}_{n_{1}} & 0 \\
0 & \alpha_{2} X_{a}^{\left(N_{2}\right)} \otimes \mathbb{1}_{n_{2}}
\end{array}\right),
$$

with low-energy gauge group $S U\left(n_{1}\right) \times S U\left(n_{2}\right) \times U(1)$.

Again, the $X_{a}^{(N)}$ are interpreted as coordinate functions of a fuzzy sphere $S_{N}^{2}$, and the "scalar" action

$$
S_{\phi}=\operatorname{Tr} V(\phi)=\operatorname{Tr}\left(a^{2}\left(\phi_{a} \phi_{a}+\tilde{b}\right)^{2}+\frac{1}{\tilde{g}^{2}} F_{a b}^{\dagger} F_{a b}\right)
$$

for $N \times N$ matrices $\phi_{a}$ is precisely the action for a $U(n)$ Yang-Mills theory on $S_{N}^{2}$ with coupling $\tilde{g}$, as shown in [31]. In fact, the new term $\left(\phi_{a} \phi_{a}+\tilde{b}\right)^{2}$ is essential for this interpretation, since it stabilizes the vacuum $\phi_{a}=X_{a}^{(N)}$ and gives a large mass to the extra "radial" scalar field which otherwise arises. The fluctuations of $\phi_{a}=X_{a}^{(N)}+A_{a}$ then provide the components $A_{a}$ of a higher-dimensional gauge field $A_{M}=\left(A_{\mu}, A_{a}\right)$, and the action can be interpreted as YM theory on the 6-dimensional space $M^{4} \times S_{N}^{2}$, with gauge group depending on the particular vacuum. We therefore interpret the vacuum as describing dynamically generated extra dimensions in the form of a fuzzy sphere $S_{N}^{2}$. This geometrical interpretation can be fully justified by working out the spectrum of Kaluza-Klein modes. The effective low-energy theory is then given by the zero modes on $S_{N}^{2}$. This approach provides a clear dynamical selection of the geometry due to the term $\left(\phi_{a} \phi_{a}+\tilde{b}\right)^{2}$ in the action.

Perhaps the most remarkable aspect of this model is that the geometric interpretation and the corresponding low-energy degrees of freedom depend in a nontrivial way on the parameters of the model, which are running under the RG group. Therefore the massless degrees of freedom and their geometrical interpretation depend on the energy scale. In particular, the low-energy gauge group generically turns out to be $S U\left(n_{1}\right) \times S U\left(n_{2}\right) \times U(1)$ or $S U(n)$, while gauge groups which are products of more than two simple components (apart from $U(1)$ ) do not seem to occur. The values of $n_{1}$ and $n_{2}$ are determined dynamically, and with the appropriate choice of parameters it is possible to construct vacuum solutions where they are as small, such as 2 and 3 [20].

It is interesting to examine the running of the coupling constants under the RG. $R$ turns out to run only logarithmically, implies that the scale of the internal spheres is only mildly affected 
by the RG flow. However, $\tilde{b}$ is running essentially quadratically, hence is generically large. This is quite welcome here: starting with some large $N, \tilde{b} \approx C_{2}(\tilde{N})$ must indeed be large in order to lead to the geometric interpretation discussed above. Hence the problems of naturalness or fine-tuning appear to be rather mild here.

A somewhat similar model has been studied in [35], which realizes deconstruction and a "twisted" compactification of an extra fuzzy sphere based on a supersymmetric gauge theory. Our model is different and does not require supersymmetry, leading to a much richer pattern of symmetry breaking and effective geometry.

The dynamical formation of fuzzy spaces found here is also related to recent work studying the emergence of stable submanifolds in modified IIB matrix models. In particular, previous studies based on actions for fuzzy gauge theory different from ours generically only gave results corresponding to $U(1)$ or $U(\infty)$ gauge groups, see e.g. [36, 37, 38] and references therein. The dynamical generation of a nontrivial index on noncommutative spaces has also been observed in $[39,40]$ for different models.

Our mechanism may also be very interesting in the context of the recent observation [41] that extra dimensions are very desirable for the application of noncommutative field theory to particle physics. Other related recent work discussing the implications of the higher-dimensional point of view on symmetry breaking and Higgs masses can be found in [42, 43, 44, 45]. These issues could now be discussed within a renormalizable framework.

\subsection{Inclusion of fermions}

So far we have only discussed the emergence of fuzzy extra dimensions in a four-dimensional and renormalizable pure Yang-Mills theory. Let us now include fermions. First we discuss the Dirac operator on the fuzzy sphere and its spectrum in the type I and type II vacua. Subsequently, we construct a model which dynamically develops fuzzy extra dimensions with the geometry of a product of two fuzzy spheres and study the zero modes of the corresponding Dirac operator in such vacua.

\subsubsection{Fermions on $M^{4} \times S^{2}$ and $M^{4} \times S_{N}^{2}$}

We first recall the classical description of fermions on $M^{4} \times S^{2}$, formulated in a way which will generalize to the fuzzy case. This is done using the embedding $S^{2} \subset \mathbb{R}^{3}$ based on the 7-dimensional Clifford algebra

$$
\Gamma^{A}=\left(\Gamma^{\mu}, \Gamma^{a}\right)=\left(\mathbb{1} \otimes \gamma^{\mu}, \sigma^{a} \otimes i \gamma_{5}\right) .
$$

Here $\sigma^{a}, a=1,2,3$ generate the $S O(3) \sim S U(2)$ Clifford algebra. The $\Gamma^{A}$ act on $\mathbb{C}^{2} \otimes \mathbb{C}^{4}$ and satisfy $\left(\Gamma^{A}\right)^{\dagger}=\eta^{A B} \Gamma^{B}$ where $\eta^{A B}=(1,-1, \ldots,-1)$ is the 7 -dimensional Minkowski metric. The corresponding 8-component spinors describe Dirac fermions on $M^{4} \times S^{2}$, and can be viewed as Dirac spinors on $M^{4}$ tensored with 2-dimensional Dirac spinors on $S^{2} \subset \mathbb{R}^{3}$. We can define a 2-dimensional chirality operator $\chi$ locally at each point of the unit sphere $S^{2}$ by setting

$$
\chi=x_{a} \sigma^{a},
$$

which has eigenvalues \pm 1 . At the north pole $x_{a}=(0,0,1)$ of $S^{2}$ this coincides with $\chi=$ $-i \sigma^{1} \sigma^{2}=\sigma^{3}$, as expected. The action for a Dirac fermion on $M^{4} \times S^{2}$ can then be written as

$$
S_{6 D}=\int_{M^{4}} d^{4} y \int_{S^{2}} d \Omega \bar{\Psi}_{D}\left(i \gamma^{\mu} \partial_{\mu}+i \gamma_{5} \not D_{(2)}+m\right) \Psi_{D},
$$

where

$$
\not D_{(2)} \Psi_{D}=\left(\sigma_{a} L_{a}+1\right) \Psi_{D}
$$


is the Dirac operator on $S^{2}$ in "global" notation and $L_{a}$ are the angular momentum operators, while the constant 1 in (3.3) ensures $\left\{\not D_{(2)}, \chi\right\}=0$ and reflects the curvature of $S^{2}$. This is equivalent to the standard formulation in terms of a comoving frame, but more appropriate for the fuzzy case.

As we discussed in Section 2.4, chiral (Weyl) spinors $\Psi_{ \pm}$on $M^{4} \times S^{2}$ are then defined using the $6 \mathrm{D}$ chirality operator

$$
\Gamma=\gamma_{5} \chi,
$$

where $\gamma_{5}$ is the four-dimensional chirality operator, and they satisfy $\Gamma \Psi_{ \pm}= \pm \Psi_{ \pm}$. They contain both chiralities from the four-dimensional point of view,

$$
\Psi_{ \pm}=(0,1 ; \pm)+(1,0 ; \mp)
$$

where $(0,1 ; \pm)$ denotes a Weyl spinor $\psi_{\alpha}$ on $M^{4}$ with eigenvalue \pm 1 of $\chi$, and $(0,1 ; \mp)$ a dotted Weyl spinor $\bar{\psi}^{\dot{\alpha}}$ on $M^{4}$ with eigenvalue $\mp 1$ of $\chi$. These components are of course mixed under the six-dimensional rotations.

Let us now collect the main facts about the "standard" Dirac operator on the fuzzy sphere [46], which is given by the following analog of (3.3)

$$
\not D_{(2)} \Psi=\sigma_{a}\left[i X_{a}, \Psi\right]+\Psi
$$

where $\left[X_{a}, X_{b}\right]=\varepsilon_{a b c} X_{c}$ generate the fuzzy sphere as explained before. Let us recall that $X_{a}$ is antihermitian here. $\not D_{(2)}$ acts on 2-component spinors

$$
\Psi=\left(\begin{array}{c}
\psi_{1} \\
\psi_{2}
\end{array}\right)
$$

For spinors in the adjoint of the gauge group, the generators $X_{a}$ are replaced by the covariant coordinates $\phi_{a}$, and the gauged Dirac operator is

$$
\not D_{(2)} \Psi=\sigma_{a}\left[i \phi_{a}, \Psi\right]+\Psi \text {. }
$$

Let us note that there does not exist a chirality operator which anticommutes with $\not_{(2)}$ and has eigenvalues \pm 1 ; this follows from the spectrum of $\not D_{(2)}$, which will be determined below.

\subsubsection{The spectrum of $\not D_{(2)}$ in a type I vacuum}

Since $\not_{(2)}$ commutes with the $S U(2)$ group of rotations, the eigenmodes of $\not_{(2)}$ in the type I vacuum are obtained by decomposing the spinors into irreps of $S U(2)$

$$
\begin{aligned}
\Psi & \in(2) \otimes(N) \otimes(N)=(2) \otimes((1) \oplus(3) \oplus \cdots \oplus(2 N-1)) \\
& =((2) \oplus(4) \oplus \cdots \oplus(2 N)) \oplus((2) \oplus \cdots \oplus(2 N-2))=:\left(\Psi_{+,(n)} \oplus \Psi_{-,(n)}\right) .
\end{aligned}
$$

This defines the spinor harmonics $\Psi_{ \pm,(n)}$ which live in the $n$-dimensional representation of $S U(2)$ denoted by $(n)$ for $n=2,4, \ldots, 2 N$, excluding $\Psi_{-,(2 N)}$. The eigenvalue of $\not_{(2)}$ acting on these states can be determined easily using some $S U(2)$ algebra [31]:

$$
\not D_{(2)} \Psi_{ \pm,(n)}=E_{\delta= \pm,(n)} \Psi_{ \pm,(n)},
$$

where

$$
E_{\delta= \pm,(n)} \approx \frac{\alpha}{2}\left\{\begin{array}{lll}
n, & \delta=1, & n=2,4, \ldots, 2 N \\
-n, & \delta=-1, & n=2,4, \ldots, 2 N-2
\end{array}\right.
$$

assuming $\alpha \approx 1$; this is exact for $\alpha=1$. We note that with the exception of $\Psi_{+,(2 N)}$, all eigenstates come in pairs $\left(\Psi_{+,(n)}, \Psi_{-,(n)}\right)$ for $n=2,4, \ldots, 2 N-2$, which have opposite eigenvalues $\pm \frac{\alpha}{2} n$ of $\not D_{(2)}$. 


\subsubsection{The spectrum of $D_{(2)}$ in a type II vacuum}

Consider now a type II vacuum,

$$
\left(\begin{array}{cc}
\alpha_{1} X_{a}^{N_{1}} \otimes \mathbb{1}_{n_{1}} & 0 \\
0 & \alpha_{2} X_{a}^{N_{2}} \otimes \mathbb{1}_{n_{2}}
\end{array}\right)
$$

We decompose the spinors according to this block-structure as

$$
\Psi=\left(\begin{array}{ll}
\Psi^{11} & \Psi^{12} \\
\Psi^{21} & \Psi^{22}
\end{array}\right)
$$

The analysis for the diagonal blocks is the same as before, and they describe fermions in the adjoint of $S U\left(n_{1}\right)$ resp. $S U\left(n_{2}\right)$. The off-diagonal blocks however describe fermions in the bifundamental $\left(n_{1}\right) \times\left(\bar{n}_{2}\right)$ of $S U\left(n_{1}\right) \times S U\left(n_{2}\right)$, and those will provide the interesting lowenergy sector. For the moment we ignore the extra $S U\left(n_{i}\right)$ structure. Assuming $N_{1} \neq N_{2}$, their decomposition (3.4) into irreps of the global $S U(2)$ now reads

$$
\begin{aligned}
\Psi^{12} \in & (2) \otimes\left(N_{1}\right) \otimes\left(N_{2}\right)=(2) \otimes\left(\left(1+\left|N_{2}-N_{1}\right|\right) \oplus\left(3+\left|N_{2}-N_{1}\right|\right) \oplus \cdots \oplus\left(N_{1}+N_{2}-1\right)\right) \\
= & \left(\left(\left|N_{2}-N_{1}\right|+2\right) \oplus\left(\left|N_{2}-N_{1}\right|+4\right) \oplus \cdots \oplus\left(N_{1}+N_{2}\right)\right) \\
& \oplus\left(\left(\left|N_{2}-N_{1}\right|\right) \oplus\left(\left|N_{2}-N_{1}\right|+2\right) \oplus \cdots \oplus\left(N_{1}+N_{2}-2\right)\right)=:\left(\Psi_{+,(n)}^{12} \oplus \Psi_{-,(n)}^{12}\right)
\end{aligned}
$$

defining the spinor harmonics $\Psi_{ \pm,(n)}^{12}$ which live in the representation $(n)$ of $S U(2)$. A similar decomposition holds for $\Psi^{21} \in(2) \otimes\left(N_{2}\right) \otimes\left(N_{1}\right)$.

\subsection{Dynamical generation of fuzzy $S^{2} \times S^{2}$ and mirror fermions}

As we have previously discussed in Section 2.4 a single fuzzy sphere is not a good candidate in order to obtain chiral fermions in four dimensions. However, a product of two fuzzy spheres [47] is more promising, since its isometry group is $S=S U(2) \times S U(2)$ with dimension $\operatorname{dim} S=6$, which is of the form $4 n+2$, as required. Therefore we shall study this case in the context of dynamical generation of fuzzy extra dimensions and explore the fermionic sector in a type II vacuum where fluxes can be included. It will turn out that the fermions are accommodated in complex, bifundamental representations which however come in pairs of opposite chirality. This picture corresponds then to mirror fermions [48].

\subsubsection{The action}

Let us consider a $S U(N)$ Yang-Mills gauge theory in four-dimensional Minkowski spacetime, coupled to six scalars $\phi_{a}=\phi_{a}^{\dagger}(a=1, \ldots, 6)$ and four Majorana spinors $\chi_{p}(p=1, \ldots, 4)$ in the adjoint representation of the $S U(N)$. Moreover, we assume that the $\phi_{a}$ transform in the vector representation of a global $S U(4) \cong S O(6)$ group and the $\chi_{p}$ in the fundamental of the $S U(4)$. The above particle spectrum coincides with the spectrum of the $\mathcal{N}=4$ supersymmetric Yang-Mills theory (SYM) [49], where the global $S U(4)$ is the $R$-symmetry of the theory. The corresponding action is given by

$$
\begin{aligned}
\mathcal{S}_{\mathrm{YM}}= & \int d^{4} x\left[\operatorname{Tr}\left(-\frac{1}{4} F_{\mu \nu} F^{\mu \nu}+\frac{1}{2} \sum_{a=1}^{6} D^{\mu} \phi_{a} D_{\mu} \phi_{a}-V(\phi)\right)\right. \\
& \left.+\frac{1}{2} \operatorname{Tr}\left(i \bar{\chi}_{p} \not D \chi_{p}+g_{4}\left(\Delta_{R}^{a}\right)_{p q} \bar{\chi}_{p} R\left[\phi_{a}, \chi_{q}\right]-g_{4}\left(\Delta_{L}^{a}\right)_{p q} \bar{\chi}_{p} L\left[\phi_{a}, \chi_{q}\right]\right)\right],
\end{aligned}
$$


where the potential has the form

$$
V(\phi)=V_{\mathcal{N}=4}(\phi)+V_{\text {break }}(\phi) .
$$

In (3.6) the first term corresponds to the potential of the $\mathcal{N}=4$ SYM theory, which is explicitly given by

$$
V_{\mathcal{N}=4}(\phi)=-\frac{1}{4} g_{4}^{2} \sum_{a, b}\left[\phi_{a}, \phi_{b}\right]^{2}
$$

while the second term corresponds to an explicit $R$-symmetry-breaking potential, which breaks the $\mathcal{N}=4$ supersymmetry as well as the global $S U(4)$ symmetry. We shall see in Section 4 that this potential actually corresponds to a set of $\mathcal{N}=1$ soft supersymmetry breaking terms, which will be explicitly presented later.

In the above expressions $\mu, \nu=0,1,2,3$ are four-dimensional spacetime indices and $D_{\mu}=$ $\partial_{\mu}-i g\left[A_{\mu}, \cdot\right]$ is the four-dimensional covariant derivative in the adjoint representation. The projection operators $L$ and $R$ are, as usual, defined as $L=\frac{1}{2}\left(\mathbb{1}-\gamma_{5}\right)$ and $R=\frac{1}{2}\left(\mathbb{1}+\gamma_{5}\right)$. The $\left(\Delta_{L}^{a}\right)_{p q}$ and $\left(\Delta_{R}^{a}\right)_{p q}$ are the intertwiners of the $\mathbf{4} \times \mathbf{4} \rightarrow \mathbf{6}$ and $\overline{\mathbf{4}} \times \overline{\mathbf{4}} \rightarrow \mathbf{6}$ respectively, namely they are Clebsch-Gordan coefficients that couple two 4 s to a $\mathbf{6}$. The Yukawa interactions in (3.5) are separately invariant under the $S U(4)$, since the $R \chi_{p}$ transforms in the $\mathbf{4}$ and the $L \chi_{p}$ in the $\overline{\mathbf{4}}$ of the $S U(4)$.

The action (3.5) without the term $V_{\text {break }}$ can be obtained by a toroidal dimensional reduction of ten-dimensional $\mathcal{N}=1 \mathrm{SYM}$ theory [49]. The corresponding ten-dimensional action is

$$
\mathcal{S}_{D=10}=-\frac{1}{4 g_{10}^{2}} \int d^{10} x \operatorname{Tr} F_{M N} F^{M N}+\frac{1}{2} \int d^{10} x \operatorname{Tr} \bar{\Psi} i \Gamma^{M} D_{M} \Psi
$$

where

$$
D_{M}=\partial_{M}-i g\left[A_{M}, \cdot\right]
$$

capital Latin letters denote ten-dimensional indices, i.e. $M=0, \ldots, 9$ and $\Psi$ is a ten-dimensional Majorana-Weyl spinor. Considering a compactification of the form $M^{4} \times Y$, the scalars are obtained from the internal components of the higher-dimensional gauge field according to the splitting

$$
A_{M}=\left(A_{\mu}, \Phi_{3+a}\right), \quad a=1, \ldots, 6 .
$$

The ten-dimensional Clifford algebra, generated by $\Gamma_{M}$, naturally separates into a four-dimensional and a six-dimensional one as follows,

$$
\Gamma_{M}=\left(\Gamma_{\mu}, \Gamma_{3+a}\right), \quad \Gamma_{\mu}=\gamma_{\mu} \otimes \mathbb{1}_{8}, \quad \Gamma_{3+a}=\gamma_{5} \otimes \Delta_{a} .
$$

Here the $\gamma_{\mu}$ define the four-dimensional Clifford algebra and they are chosen to be purely imaginary, corresponding to the Majorana representation in four dimensions (see Appendix A), while the $\Delta_{a}$ define the six-dimensional Euclidean Clifford algebra and they are chosen to be real and antisymmetric. Then it is straightforward to see that $\gamma_{0}=\gamma_{0}^{\dagger}=-\gamma_{0}^{T}$ and $\gamma_{i}=-\gamma_{i}^{\dagger}=\gamma_{i}^{T}$. The ten-dimensional chirality operator is

$$
\Gamma^{(11)}=\gamma_{5} \otimes \Gamma^{(Y)},
$$

where the four- and six-dimensional chirality operators are defined as

$$
\gamma_{5}=-i \gamma_{0} \cdots \gamma_{3}=\gamma_{5}^{\dagger}=-\gamma_{5}^{T},
$$




$$
\Gamma^{(Y)}=-i \Delta_{1} \cdots \Delta_{6}=\left(\Gamma^{(Y)}\right)^{\dagger}=-\left(\Gamma^{(Y)}\right)^{T}
$$

Let us denote the ten-dimensional charge conjugation operator as

$$
\mathcal{C}=C^{(4)} \otimes C^{(6)}
$$

where $C^{(4)}$ is the four-dimensional charge conjugation operator and $C^{(6)}=\mathbb{1}_{8}$ in our conventions. This operator satisfies, as usual, the relation

$$
\mathcal{C} \Gamma^{M} \mathcal{C}^{-1}=-\left(\Gamma^{M}\right)^{T}
$$

Then the Majorana-Weyl condition in ten dimensions is ${ }^{6}$

$$
\Psi^{C}=\mathcal{C} \bar{\Psi}^{T}=\Psi
$$

where

$$
\bar{\Psi}=\Psi^{T} \mathcal{C}^{T}, \quad \Psi^{\dagger}=\Psi^{T} \mathcal{C}^{T} \gamma_{0}=\Psi^{T}
$$

Let us note that in the Majorana representation, where the $\gamma_{\mu}$ are imaginary, the four-dimensional charge conjugation operator is $C^{(4)}=-\gamma_{0}$.

Performing a trivial dimensional reduction from ten to four dimensions, i.e. assuming that all fields do not depend on the internal coordinates, it is well-known that the Yang-Mills part of the ten-dimensional action leads to the bosonic part of the $\mathcal{N}=4 \mathrm{SYM}$ in four dimensions, as in (3.5), with the potential term having the form (3.7). The couplings $g_{4}$ and $g_{10}$ are related through the volume $V$ of the internal six-dimensional torus as $g_{4}=\frac{g_{10}}{\sqrt{V}}$.

The reduction of the Dirac term is performed similarly. The Majorana-Weyl spinor $\Psi$ has the form

$$
\Psi=\sum_{p=1}^{4}\left(R \chi_{p} \otimes \eta_{p}+L \chi_{p} \otimes \eta_{p}^{*}\right), \quad \bar{\Psi}=\sum_{p=1}^{4}\left(\bar{\chi}_{p} L \otimes \eta_{p}^{\dagger}+\bar{\chi}_{p} R \otimes \eta_{p}^{T}\right)
$$

where the $\chi_{p}$ are four-dimensional Majorana spinors and the $\eta_{p}$ are the four complex eigenvectors of the $\Gamma^{(Y)}$ with eigenvalue +1 . Since the $\Gamma^{(Y)}$ is purely imaginary the $\eta_{p}^{*}$ have eigenvalue -1 . Assuming that the spinor is independent of the extra-dimensional coordinates, the dimensional reduction of the Dirac term of the ten-dimensional action leads in four dimensions to the kinetic term for the spinor $\chi_{p}$ and the Yukawa couplings as they appear in (3.5). In particular, the Yukawa couplings arise from the term

$$
\operatorname{Tr} \bar{\Psi} i \not D_{(6)} \Psi=\operatorname{Tr} \bar{\Psi} \Delta^{a}\left[\Phi_{a}, \Psi\right]
$$

where $\not D_{(6)}$ denotes the Dirac operator on the internal space, which satisfies

$$
\left\{\not D_{(6)}, \Gamma^{(Y)}\right\}=0
$$

and it will be related in the ensuing to the effective Dirac operator on the fuzzy extra dimensions.

\footnotetext{
${ }^{6}$ Note that $T$ transposes only the spinor.
} 


\subsubsection{Type I vacuum and fuzzy $S^{2} \times S^{2}$}

We now assume that the renormalizable potential in the four-dimensional action admits vacua corresponding to the product of two fuzzy spheres, i.e.

$$
\begin{aligned}
& \phi_{i}^{L} \equiv \phi_{i}=\alpha_{L} \lambda_{i}^{\left(N_{L}\right)} \otimes \mathbb{1}_{N_{R}} \otimes \mathbb{1}_{n}, \\
& \phi_{i}^{R} \equiv \phi_{3+i}=\alpha_{R} \mathbb{1}_{N_{L}} \otimes \lambda_{i}^{\left(N_{R}\right)} \otimes \mathbb{1}_{n}, \quad i=1,2,3,
\end{aligned}
$$

where $\lambda_{i}^{(N)}$ denotes the generator of the $N$-dimensional irreducible representation of $S U(2)$ and therefore

$$
\left[\phi_{i}^{L}, \phi_{j}^{L}\right]=i \alpha_{L} \varepsilon_{i j k} \phi_{k}^{L}, \quad \phi_{i}^{L} \phi_{i}^{L}=\alpha_{L}^{2} \frac{N_{L}^{2}-1}{4},
$$

and similarly for the $\phi_{i}^{R}$. Moreover the two algebras commute with each other, i.e.

$$
\left[\phi_{i}^{L}, \phi_{j}^{R}\right]=0 .
$$

The vacuum (3.8) can be obtained by choosing the potential $V(\Phi)$ to have the following form,

$$
V[\Phi]=a_{L}^{2}\left(\phi_{i}^{L} \phi_{i}^{L}+b_{L} \mathbb{1}\right)^{2}+a_{R}^{2}\left(\phi_{i}^{R} \phi_{i}^{R}+b_{R} \mathbb{1}\right)^{2}+\frac{1}{g_{L}^{2}} F_{i j}^{L} F_{i j}^{L}+\frac{1}{g_{R}^{2}} F_{i j}^{R} F_{i j}^{R},
$$

where

$$
F_{i j}^{L}=\left[\phi_{i}^{L}, \phi_{j}^{L}\right]-i \varepsilon_{i j k} \phi_{k}^{L}, \quad F_{i j}^{R}=\left[\phi_{i}^{R}, \phi_{j}^{R}\right]-i \varepsilon_{i j k} \phi_{k}^{R},
$$

which will be interpreted as field strengths on the spontaneously generated fuzzy spheres, as in Section 3.2. The potential (3.9) breaks the global $S O(6)$ symmetry down to $S O(3)_{L} \times S O(3)_{R}$ and for suitable parameters $a_{L / R}, b_{L / R}, g_{L / R}$, its stable global minimum is indeed given by (3.8) up to $U(N)$ gauge transformations, provided that

$$
N=N_{L} N_{R} n
$$

Such a vacuum should be interpreted as a stack of $n$ fuzzy branes with geometry $S_{L}^{2} \times S_{R}^{2}$ and in the present construction it breaks the gauge group $S U(N)$ down to $S U(n)$.

\subsubsection{Operators on $S_{L}^{2} \times S_{R}^{2}$}

Having in mind a compactification on $S_{L}^{2} \times S_{R}^{2} \subset \mathbb{R}^{6}$, we organize the internal $S O(6)$ structure according to its subgroup $S O(3)_{L} \times S O(3)_{R}$. Then, if $\Delta_{a}$ define the six-dimensional Euclidean Clifford algebra (see Appendix A) it is natural to adopt the notation

$$
\Delta_{i}^{L}=\Delta_{i}, \quad \Delta_{i}^{R}=\Delta_{3+i}, \quad i=1,2,3 .
$$

Let us consider the following $S O(3)_{L} \times S O(3)_{R}$ invariant operators on each sphere [47],

$$
\chi_{L}=\frac{i}{2 R_{L}} \Delta_{i}^{L}\left\{\phi_{i}^{L}, \cdot\right\} \sim \frac{i}{R_{L}} \Delta_{L}^{i} x_{i}^{L}, \quad \chi_{L, \operatorname{tang}}=\Gamma_{L}^{(Y)} \chi_{L}, \quad \Gamma_{L}^{(Y)}=\Delta_{1} \Delta_{2} \Delta_{3},
$$

where

$$
R_{L}=\alpha_{L} N_{L}
$$


denotes the radius of $S_{L}^{2}$ and the operators $\chi_{R}, \chi_{R \text {,tang }}$ and $\Gamma_{R}^{(Y)}$ are defined similarly. Here $\sim$ denotes the semi-classical limit, i.e. the limit $N \rightarrow \infty$. These operators are hermitian, and they satisfy the relations

$$
\begin{aligned}
& \left\{\chi_{L / R}, \Gamma^{(Y)}\right\}=\left[\chi_{L} \chi_{R}, \Gamma^{(Y)}\right]=0, \quad\left\{\chi_{L}, \chi_{R}\right\}=0, \\
& {\left[\chi_{L, \text { tang }}, \chi_{R, \text { tang }}\right]=0, \quad \chi_{L / R}^{2} \sim \mathbb{1} \sim \chi_{L / R, \operatorname{tang}}^{2}}
\end{aligned}
$$

in a $S_{L}^{2} \times S_{R}^{2}$ vacuum (3.8). In order to understand their meaning, let us consider $S_{L}^{2}$. On the north pole with $x_{1} \sim 0, x_{2} \sim 0, x_{3} \sim R_{L}$, the tangential chirality operator is given by $\chi_{L \text {,tang }} \sim i \Delta_{1} \Delta_{2}$, while the operator $\chi_{L} \sim i \Delta_{3}$ is perpendicular. Therefore the $S U(2)_{L} \times S U(2)_{R^{-}}$ invariant operator

$$
\chi^{\perp}:=i \chi_{L} \chi_{R},
$$

which squares to one, $\left(\chi^{\perp}\right)^{2} \sim 1$, corresponds to the chirality operator on the two-dimensional space which is perpendicular to $S_{L}^{2} \times S_{R}^{2} \subset \mathbb{R}^{6}$. In addition, the operator

$$
\chi_{\text {tang }}:=\Gamma^{(Y)} \chi^{\perp}=-\chi_{L, \operatorname{tang}} \chi_{R, \text { tang }} \sim \Delta_{1} \Delta_{2} \Delta_{4} \Delta_{5}, \quad\left(\chi_{\text {tang }}\right)^{\dagger}=\chi_{\text {tang }}
$$

is the tangential chirality operator on $S_{L}^{2} \times S_{R}^{2}$.

In order to understand the fuzzy Kaluza-Klein modes, it is important to understand the relation of the Dirac operator on the internal space, let us call it $\not D_{(6)}$, with the fuzzy Dirac operators on $S_{L}^{2}$ and $S_{R}^{2}$. We note that in the Majorana representation of the six-dimensional Clifford algebra, we have

$$
-i \Gamma_{L}^{(Y)} \Delta_{i}^{L}=\mathbb{1}_{2} \otimes \gamma_{L}^{i},
$$

where $\gamma_{L}^{i}=U^{-1}\left(\sigma_{i} \otimes \mathbb{1}_{2}\right) U$ is essentially a double-degenerate representation of the threedimensional Clifford algebra. This allows to write

$$
\Delta_{L}^{i}\left[\phi_{i}^{L}, \Psi\right]+i \alpha_{L} \Gamma_{L}^{(Y)} \Psi=i \Gamma_{L}^{(Y)} \not D_{S_{L}^{2}} \Psi
$$

where $\not D_{S_{L}^{2}}$ is the standard Dirac operator on the fuzzy $S_{L}^{2}$, as described in Section 3.3.1. Note that one usually works with two-component spinors on the fuzzy sphere, where the tangential chirality operator is given by $\sigma_{1} \sigma_{2}=i \sigma_{3}$. Here $\Delta_{3}$ is independent of $\Delta_{1} \Delta_{2}$ and therefore $\chi_{L \text {,tang }}$ is the proper tangential chirality operator on the $S_{L}^{2}$, rather than $\chi_{L}$. We thus obtain the relation of $\not D_{(6)}$ with a "tangential" Dirac operator on $S^{2} \times S^{2} \subset \mathbb{R}^{6}$ :

$$
\not D_{(6)}=\not D_{S^{2} \times S^{2}}-\alpha_{L} \Gamma_{L}^{(Y)}-\alpha_{R} \Gamma_{R}^{(Y)},
$$

where

$$
\not D_{S^{2} \times S^{2}}=\Gamma_{L}^{(Y)} \not D_{S_{L}^{2}}+\Gamma_{R}^{(Y)} \not D_{S_{R}^{2}} .
$$

Then the term

$$
\int \bar{\Psi} i \not D_{(6)} \Psi
$$

which gives rise to the Yukawa terms in the action (3.5), becomes

$$
\mathcal{S}_{y u k}=\int \bar{\Psi} i \not D_{S^{2} \times S^{2}} \Psi+\mathcal{S}_{\text {shift }},
$$

where the shift action

$$
\mathcal{S}_{\text {shift }}=\int i \operatorname{Tr} \bar{\Psi} \gamma_{5}\left(\alpha_{L} \Gamma_{L}^{(Y)}+\alpha_{R} \Gamma_{R}^{(Y)}\right) \Psi
$$

is recognized as curvature effect. One can show that $\not D_{S^{2} \times S^{2}}$ reduces in the semi-classical limit to the Dirac operator on $S_{L}^{2} \times S_{R}^{2}$ in the above background geometry (3.8) [47]. 


\subsubsection{Type II vacuum and the zero-modes}

In order to obtain massless fermions, it is necessary to add magnetic fluxes $m_{L}, m_{R}$ on the two spheres. As explained previously, this is realized in a slightly modified class of vacua, called "type II vacua". In the present case such a vacuum has the form

$$
\begin{aligned}
& \phi_{i}=\left(\begin{array}{cc}
\alpha_{1} \lambda_{i}^{\left(N_{L}^{1}\right)} \otimes \mathbb{1}_{N_{R}^{1}} \otimes \mathbb{1}_{n_{1}} & 0 \\
0 & \alpha_{2} \lambda_{i}^{\left(N_{L}^{2}\right)} \otimes \mathbb{1}_{N_{R}^{2}} \otimes \mathbb{1}_{n_{2}}
\end{array}\right), \\
& \phi_{3+i}=\left(\begin{array}{cc}
\alpha_{3} \mathbb{1}_{N_{L}^{1}} \otimes \lambda_{i}^{\left(N_{R}^{1}\right)} \otimes \mathbb{1}_{n_{1}} & 0 \\
0 & \alpha_{4} \mathbb{1}_{N_{L}^{2}} \otimes \lambda_{i}^{\left(N_{R}^{2}\right)} \otimes \mathbb{1}_{n_{2}}
\end{array}\right), \quad i=1,2,3 .
\end{aligned}
$$

The commutant of these generators, i.e. the unbroken gauge group, is $S U\left(n_{1}\right) \times S U\left(n_{2}\right) \times U(1)_{Q}$, where the $U(1)_{Q}$ has generator

$$
Q=\left(\begin{array}{cc}
\frac{1}{N_{R}^{1} N_{L}^{1} n_{1}} \mathbb{1} & 0 \\
0 & -\frac{1}{N_{R}^{2} N_{L}^{2} n_{2}} \mathbb{1}
\end{array}\right) .
$$

This vacuum corresponds to a splitting

$$
N=n_{1} N_{L}^{1} N_{R}^{1}+n_{2} N_{L}^{2} N_{R}^{2},
$$

which is more generic than (3.10). It determines a splitting of the fermionic wavefunction

$$
\Psi=\left(\begin{array}{ll}
\Psi^{11} & \Psi^{12} \\
\Psi^{21} & \Psi^{22}
\end{array}\right)
$$

where $\Psi^{12}$ transforms in the bifundamental representation $\left(n_{1}\right) \otimes\left(\bar{n}_{2}\right)$ of the $S U\left(n_{1}\right) \times S U\left(n_{2}\right)$ and $\Psi^{21}$ in the $\left(\bar{n}_{1}\right) \otimes\left(n_{2}\right)$. The Majorana condition $\Psi^{+} \equiv \Psi^{\dagger T}=\Psi$ implies

$$
\left(\Psi^{11}\right)^{+}=\Psi^{11}, \quad\left(\Psi^{22}\right)^{+}=\Psi^{22}, \quad\left(\Psi^{12}\right)^{+}=\Psi^{21} .
$$

The interpretation of this vacuum is as a stack of $n_{1}$ fuzzy branes and a stack of $n_{2}$ fuzzy branes with geometry $S_{L}^{2} \times S_{R}^{2}$. However, these fuzzy spheres carry magnetic flux under the unbroken $U(1)_{Q}$ given by [31]

$$
m_{L}=N_{L}^{1}-N_{L}^{2}, \quad m_{R}=N_{R}^{1}-N_{R}^{2},
$$

on $S_{L}^{2}$ and $S_{R}^{2}$ respectively. Since the fermions $\Psi$ transform in the adjoint representation, the diagonal components $\Psi^{11}$ and $\Psi^{22}$ are unaffected, but the off-diagonal components $\Psi^{12}$ and $\Psi^{21}$ feel this magnetic flux and develop chiral zero modes according to the index theorem. This can also be seen very explicitly in the fuzzy case [21]. For example, a flux $m_{L}>0$ on $S_{L}^{2}$ implies that there are (would-be) zero modes $\Psi_{\left(m_{L}\right)}^{12}$ for $\not_{S_{L}^{2}}$ with $\chi_{L \text {,tang }}=+1$, and $\Psi_{\left(m_{R}\right)}^{21}$ with $\chi_{L, \text { tang }}=-1$.

To be specific, assume that $m_{L}>0$ and $m_{R}>0$. Then there exist ("would-be", approximate) zero modes $\Psi_{\left(m_{L}, m_{R}\right)}^{12}$ of both $\not D_{S_{L}^{2}}$ and $\not D_{S_{R}^{2}}$ and therefore of $\not_{S^{2} \times S^{2}}$, with definite chirality ${ }^{7}$

$$
\chi_{L, \operatorname{tang}} \Psi_{\left(m_{L}, m_{R}\right)}^{12}=\Psi_{\left(m_{L}, m_{R}\right)}^{12}=\chi_{R, \operatorname{tang}} \Psi_{\left(m_{L}, m_{R}\right)}^{12},
$$

\footnotetext{
${ }^{7}$ To simplify the notation we assume that the operators $\chi, \not D_{S^{2}}$ are defined appropriately such that these relations hold exactly. Otherwise the stated eigenvalues of $\chi$ and $\not_{S^{2}}$ are approximate up to $O\left(\frac{1}{N}\right)$ corrections. Since we are mainly interested in the structure of the would-be zero modes, we do not keep track of these $O\left(\frac{1}{N}\right)$ corrections here.
} 


$$
\chi_{\operatorname{tang}} \Psi_{\left(m_{L}, m_{R}\right)}^{12}=-\Psi_{\left(m_{L}, m_{R}\right)}^{12}
$$

There are also the "conjugate" zero modes $\Psi_{\left(m_{L}, m_{R}\right)}^{21}$, which satisfy

$$
\begin{aligned}
& \chi_{L, \operatorname{tang}} \Psi_{\left(m_{L}, m_{R}\right)}^{21}=-\Psi_{\left(m_{L}, m_{R}\right)}^{21}=\chi_{R, \operatorname{tang}} \Psi_{\left(m_{L}, m_{R}\right)}^{21}, \\
& \chi_{\operatorname{tang}} \Psi_{\left(m_{L}, m_{R}\right)}^{21}=-\Psi_{\left(m_{L}, m_{R}\right)}^{21} .
\end{aligned}
$$

All the other Kaluza-Klein modes have both chiralities and acquire a mass due to $\not D_{S^{2} \times S^{2}}$.

Motivated by the properties of the zero modes which are encoded in (3.11) and (3.12) let us now define the following operators,

$$
\Pi_{L} \Psi:=\gamma_{5} \chi_{L, \operatorname{tang}} \Psi, \quad \Pi_{R} \Psi:=\gamma_{5} \chi_{R, \operatorname{tang}} \Psi
$$

which satisfy $\Pi_{L}^{2} \sim \mathbb{1} \sim \Pi_{R}^{2}$. They are clearly compatible with the ten-dimensional Weyl condition and also with the ten-dimensional Majorana condition $\Psi^{\dagger}=\Psi^{T}$. Consequently they are well-defined and as we shall exhibit in the following they will select the chiral sectors of our model. In order to understand the qualitative structure of the zero modes, in particular their chirality from the four-dimensional point of view, it is enough to consider the semi-classical limit. On the north pole we have,

$$
\begin{aligned}
& \chi_{L, \text { tang }} \sim i \Delta_{1} \Delta_{2}=\mathbb{1} \otimes \sigma^{3} \otimes \sigma^{2}, \quad \chi_{R, \text { tang }} \sim i \Delta_{4} \Delta_{5}=\mathbb{1} \otimes \sigma^{2} \otimes \sigma^{3}, \\
& \chi_{\text {tang }} \sim \mathbb{1} \otimes \sigma^{1} \otimes \sigma^{1}, \quad \chi^{\perp} \sim \sigma_{2} \otimes \sigma^{1} \otimes \sigma^{1} .
\end{aligned}
$$

Then, the unique solution of (3.11) has the form

$$
\Psi_{\left(m_{L}, m_{R}\right)}^{12} \sim\left(\begin{array}{l}
\rho^{12} \\
\eta^{12}
\end{array}\right) \otimes\left(\left(\begin{array}{l}
1 \\
1
\end{array}\right) \otimes\left(\begin{array}{l}
1 \\
1
\end{array}\right)-i\left(\begin{array}{l}
1 \\
-1
\end{array}\right) \otimes\left(\begin{array}{l}
1 \\
-1
\end{array}\right)\right)
$$

where $\rho^{12}, \eta^{12}$ are four-dimensional Dirac spinors. Similarly, the unique solution of (3.12) has the form

$$
\Psi_{\left(m_{L}, m_{R}\right)}^{21} \sim\left(\begin{array}{l}
\rho^{21} \\
\eta^{21}
\end{array}\right) \otimes\left(\left(\begin{array}{l}
1 \\
1
\end{array}\right) \otimes\left(\begin{array}{l}
1 \\
1
\end{array}\right)+i\left(\begin{array}{l}
1 \\
-1
\end{array}\right) \otimes\left(\begin{array}{l}
1 \\
-1
\end{array}\right)\right) .
$$

The Weyl condition $\Gamma^{(11)} \Psi=\Psi$ implies

$$
\gamma_{5} \Psi=\Gamma^{(Y)} \Psi=-\left(\sigma_{2} \otimes \mathbb{1} \otimes \mathbb{1}\right) \Psi, \quad i \eta^{12}=\gamma_{5} \rho^{12}, \quad i \eta^{21}=\gamma_{5} \rho^{21},
$$

so that the would-be zero mode reduces essentially to

$$
\Psi_{(m)}^{12} \sim\left(\begin{array}{l}
\rho^{12} \\
-i \gamma_{5} \rho^{12}
\end{array}\right), \quad \overline{\Psi_{(m)}^{12}} \sim\left(\overline{\rho^{12}},-i \overline{\rho^{12}} \gamma_{5}\right)
$$

dropping the remaining tensor factors in (3.14). The Majorana condition, $\Psi^{+}:=\Psi^{\dagger T}=\Psi$, in the present representation implies

$$
\rho^{12}=\left(\rho^{21}\right)^{+}
$$

and it relates the upper-diagonal and lower-diagonal components. This amounts to a single four-dimensional Dirac spinor $\rho^{12}$ and the model is non-chiral. However, since the fermions transform in complex, bifundamental representations of the gauge group the model does not have a vectorlike structure. The resulting structure corresponds to that of mirror fermions [48]. 
Let us recall that we have two fuzzy spheres with fluxes and we have assumed already that $m_{L}>0$ and $m_{R}>0$. Then, the relations (3.11) and (3.12) can be written as

$$
\left.\chi_{L, \operatorname{tang}}\right|_{\Psi^{12}}=\left.\chi_{R, \operatorname{tang}}\right|_{\Psi^{12}}=+1,\left.\quad \chi_{L, \operatorname{tang}}\right|_{\Psi^{21}}=\left.\chi_{R, \operatorname{tang}}\right|_{\Psi^{21}}=-1 .
$$

It follows from (3.11) and (3.12) that the operators $\Pi_{L}$ and $\Pi_{R}$, defined in (3.13), actually coincide on the space of zero modes. Hence the full fermionic Hilbert space can be separated in two sectors as follows,

$$
\mathcal{H}_{+}=\left\{\Psi ; \Pi_{L} \Psi=\Psi\right\} \quad \text { and } \quad \mathcal{H}_{-}=\left\{\Psi ; \Pi_{L} \Psi=-\Psi\right\} .
$$

Then it is clear that $\Psi^{12}$ and $\Psi^{21}$ have opposite four-dimensional chirality in each sector. Therefore we end up with two exactly chiral mirror sectors, which are separated according to (3.15).

Therefore our result is that even though the fluxes on $S^{2} \times S^{2}$ lead indeed to the expected zero modes, the model nevertheless turns out to be non-chiral a priori. More precisely, we find essentially mirror models, where two chiral sectors arise with opposite chirality. This means that each would-be zero mode from $\Psi^{12}$ has a mirror partner from $\Psi^{21}$, with opposite chirality and gauge quantum numbers. The reason for this is that the fuzzy geometry is four-dimensional but in some sense embedded in six extra dimensions. The missing two ("shadow") dimensions are reflected in extra components of the spinors, which do not see the flux and respectively the chirality on $S^{2} \times S^{2}$. This is a crucial difference of our model comparing with models based on commutative extra dimensions, where chiral Lagrangians are easier to obtain (see $[4,7]$ and the discussion in Section 2.4 of the present paper). Thus we arrive essentially at a picture of mirror fermions discussed e.g. in [48] from a phenomenological point of view. While this may still be physically interesting since the mirror fermions may have larger mass than the ones we see at low energies, it would be desirable to find a chiral version with similar features. We shall show in the following section that using orbifold techniques this can indeed be achieved.

\section{Orbifolds, fuzzy extra dimensions and chiral models}

In the previous section we discussed that a toroidal dimensional reduction of a ten-dimensional $\mathcal{N}=1 \mathrm{SYM}$ theory to four dimensions leads to $\mathcal{N}=4$ supersymmetry in four dimensions, which is not phenomenologically acceptable mainly because it is impossible to accommodate chiral fermions in the theory. The obvious way to obtain $\mathcal{N}=1$ four-dimensional models, which might be realistic since they admit chiral fermions, is to reduce the theory on suitable manifolds such as Calabi-Yau manifolds [50] or manifolds with an $S U(3)$-structure (see, e.g. [51, 52]). However, another remarkable way to achieve $\mathcal{N}=1$ supersymmetry in four dimensions is to perform a reduction on an orbifold $[53,54]$.

In this section, in order to pursue further the possibility to obtain chiral low-energy theories within the framework of gauge theories with fuzzy extra dimensions, we shall introduce an orbifold structure similar to the one used in [55]. The authors of [55], motivated by the celebrated duality between four-dimensional $\mathcal{N}=4, U(N)$ SYM theory and Type IIB string theory on $A d S_{5} \times S^{5}$ [56], used orbifold techniques similar to [57, 58] to break some of the four supersymmetries. Considering different embeddings of a $\mathbb{Z}_{3}$ discrete group in the $R$-symmetry group of the $\mathcal{N}=4 \mathrm{SYM}$ theory and performing an orbifold projection of the original theory they determined $\mathcal{N}=0,1,2$ theories, i.e. with reduced supersymmetry. Moreover, the initial gauge group $S U(3 N)$ (realised on $3 N D 3$ branes) is broken down to $S U(N)^{3}$ and the fermions are accommodated in chiral representations of the gauge group.

\section{$4.1 \mathcal{N}=4 \mathrm{SYM}$ and $\mathbb{Z}_{3}$ orbifolds}

In this section we review the basics of the $\mathbb{Z}_{3}$ orbifold projection of the $\mathcal{N}=4$ Supersymmetric Yang-Mills (SYM) theory [49]. In particular we discuss the action of the discrete group on the 
various fields of the theory and the resulting superpotential of the projected theory.

Before introducing the orbifold projection, the theory under consideration is the $\mathcal{N}=4$ supersymmetric $S U(3 N)$ gauge theory ${ }^{8}$. This theory contains, in $\mathcal{N}=1$ language, a $S U(3 N)$ vector supermultiplet and three adjoint chiral supermultiplets $\Phi^{i}, i=1,2,3$. The component fields are the $S U(3 N)$ gauge bosons $A_{\mu}, \mu=1, \ldots, 4$, six adjoint real scalars ${ }^{9} \phi^{a}, a=1, \ldots, 6$, transforming as 6 under the $S U(4)_{R} R$-symmetry of the theory and four adjoint Weyl fermions $\psi^{p}$, $p=1, \ldots, 4$, transforming as $\mathbf{4}$ under the $S U(4)_{R}$. The theory is defined on the Minkowski spacetime, whose coordinates are denoted as $x^{\mu}, \mu=1, \ldots, 4$.

In order to discuss orbifolds we have to consider the discrete group $\mathbb{Z}_{3}$ generically as a subgroup of $S U(4)_{R}$. There are three possibilities here, which have a direct impact on the amount of remnant supersymmetry [55]:

1. $\mathbb{Z}_{3}$ is maximally in $S U(4)_{R}$, in which case we are generically led to non-supersymmetric models;

2. $\mathbb{Z}_{3}$ is embedded in an $S U(3)$ subgroup of the full $R$-symmetry group, leading to $\mathcal{N}=1$ supersymmetric models with $R$-symmetry $U(1)_{R}$;

3. $\mathbb{Z}_{3}$ is embedded in a specific $S U(2)$ subgroup of $S U(4)_{R}$, in which case the remaining supersymmetry is $\mathcal{N}=2$ with $R$-symmetry $S U(2)_{R}$.

Let us next discuss in more detail the case where $\mathbb{Z}_{3}$ is embedded in $S U(3)$, that leads to $\mathcal{N}=1$ supersymmetric models. In order to proceed we consider a generator $g \in \mathbb{Z}_{3}$. This generator is conveniently labeled (see [58]) by three integers $\vec{a} \equiv\left(a_{1}, a_{2}, a_{3}\right)$ which satisfy the condition $a_{1}+a_{2}+a_{3} \equiv 0 \bmod 3$. This condition is equivalent to the statement that the discrete group is indeed embedded in $S U(3)$ and therefore it reflects the fact that $\mathcal{N}=1$ supersymmetry is preserved.

The $\mathbb{Z}_{3}$ acts non-trivially on the various fields of the theory depending on their transformation properties under the $R$-symmetry. The geometric action of the $\mathbb{Z}_{3}$ rotation on the gauge and the gaugino fields is trivial, since they are singlets under $S U(4)_{R}$. On the other hand, the action of $\mathbb{Z}_{3}$ on the complex scalars is specified by the matrix $\gamma(g)_{i j}=\delta_{i j} \omega^{a_{i}}$, where $\omega=e^{\frac{2 \pi i}{3}}$, while the corresponding action on the fermions $\psi^{i}$ is given by $\gamma(g)_{i j}=\delta_{i j} \omega^{b_{i}}$, where ${ }^{10} b_{i}=$ $-\frac{1}{2}\left(a_{i+1}+a_{i+2}-a_{i}\right)$. In the case under study the three integers have the values $\vec{a}=(1,1,-2)$, which implies $b_{i}=a_{i}$.

However, since the matter fields also transform non-trivially under the gauge group, the discrete group acts on their gauge indices too. The action of this rotation can be described by the matrix

$$
\gamma_{3}=\left(\begin{array}{ccc}
\mathbb{1}_{N} & 0 & 0 \\
0 & \omega \mathbb{1}_{N} & 0 \\
0 & 0 & \omega^{2} \mathbb{1}_{N}
\end{array}\right) .
$$

Let us note that in general the blocks of this matrix could have different dimensionality (see, e.g. $[59,60,61])$, However, anomaly freedom of the projected theory typically requires that the dimension of the three blocks is the same as will become obvious in the following. There is an interesting exception to this rule which will be discussed in Section 4.4.1.

In order to derive the projected theory under the orbifold action, one has to keep the fields which are invariant under the combined action of the discrete group on the geometry and on the gauge indices [58]. For the gauge bosons the relevant projection is

$$
A_{\mu}=\gamma_{3} A_{\mu} \gamma_{3}^{-1} \text {. }
$$

\footnotetext{
${ }^{8}$ The gauge group is taken to be $S U(3 N)$ for notational convenience as it will be clear in the following.

${ }^{9}$ In the following we shall often work with the three complex scalars $\phi^{i}, i=1,2,3$, which correspond to the complexification of the six real ones.

${ }^{10}$ This relation is of course also understood modulo 3 .
} 
Therefore, in view of (4.1), the gauge group $S U(3 N)$ of the original theory is broken down to $H=S U(N) \times S U(N) \times S U(N)$ in the projected theory.

For the complex scalars, which transform non-trivially both under the gauge group and the $R$-symmetry, the projection is

$$
\phi_{I J}^{i}=\omega^{I-J+a_{i}} \phi_{I J}^{i}
$$

where $I, J$ are gauge indices. This means that $J=I+a_{i}$ and therefore it is easy to see that the fields which survive the orbifold projection have the form $\phi_{I, I+a_{i}}$ and they transform under the gauge group $H$ as

$$
3 \cdot((N, \bar{N}, 1)+(\bar{N}, 1, N)+(1, N, \bar{N})) .
$$

For the fermions the situation is practically the same. More specifically in this case the relevant projection is

$$
\psi_{I J}^{i}=\omega^{I-J+b_{i}} \psi_{I J}^{i} .
$$

Then the surviving fermions have the form $\psi_{I, I+b_{i}}^{i}$ and they transform under $H$ in the representations (4.2), exactly as the scalars. This is just another manifestation of the $\mathcal{N}=1$ remnant supersymmetry. Moreover, the structure of the representations (4.2) guarantees that the resulting theory does not suffer from any gauge anomalies ${ }^{11}$.

Let us next note two important features of the projected theory. First the fermions transform in chiral representations of the gauge group. Indeed, the representations (4.2) are complex bifundamental ones, and their complex conjugates do not appear in the projected theory. Secondly, there are three fermionic generations in the theory. This is expected since as we noted before the theory contains three chiral supermultiplets under $\mathcal{N}=1$, leading to three generations.

Concerning the interactions among the fields of the projected theory, let us consider the superpotential of the $\mathcal{N}=4$ supersymmetric Yang-Mills theory, which has the form [49]:

$$
W_{\mathcal{N}=4}=\epsilon_{i j k} \operatorname{Tr}\left(\Phi^{i} \Phi^{j} \Phi^{k}\right)
$$

where the three chiral superfields of the theory appear. Clearly, the superpotential after the orbifold projection has the same form but it encodes only the interactions among the surviving fields of the resulting $\mathcal{N}=1$ theory. Therefore it can be written as

$$
W_{\mathcal{N}=1}^{(\text {proj })}=\sum_{I} \epsilon_{i j k} \Phi_{I, I+a_{i}}^{i} \Phi_{I+a_{i}, I+a_{i}+a_{j}}^{j} \Phi_{I+a_{i}+a_{j}, I}^{k},
$$

where the relation $a_{1}+a_{2}+a_{3} \equiv 0 \bmod 3$ was taken into account.

\subsection{Twisted fuzzy spheres}

In the present section we introduce the "twisted fuzzy sphere" $\tilde{S}_{N}^{2}$, which is a variant of the ordinary fuzzy sphere [24] compatible with the orbifolding. It is defined by the following relations

$$
\left[\phi^{i}, \phi^{j}\right]=i \epsilon_{i j k}\left(\phi^{k}\right)^{\dagger}, \quad \phi^{i}\left(\phi^{i}\right)^{\dagger}=R^{2},
$$

where $\left(\phi^{i}\right)^{\dagger}$ denotes hermitean conjugation of the complex scalar field $\phi^{i}$ and $\left[R^{2}, \phi^{i}\right]=0$. The relation (4.4) is compatible with the $\mathbb{Z}_{3}$ group action, in contrast to the usual fuzzy sphere.

\footnotetext{
${ }^{11}$ On the contrary, had we considered that the matrix (4.1) contained blocks of different dimensionality the projected theory would be anomalous and therefore additional sectors would be necessary in order to cancel the gauge anomalies.
} 
Indeed, a quick look at equation (4.2) reveals that the scalar fields are expected to satisfy (4.4) instead of the commutation relations for the ordinary fuzzy sphere. Nevertheless the above relations are closely related to a fuzzy sphere. This can be seen by considering the untwisted fields $\tilde{\phi}_{i}$, defined by

$$
\phi^{i}=\Omega \tilde{\phi}^{i},
$$

for some $\Omega \neq 1$ which satisfies

$$
\Omega^{3}=1, \quad\left[\Omega, \phi^{i}\right]=0, \quad \Omega^{\dagger}=\Omega^{-1}
$$

$\operatorname{and}^{12}$

$$
\left(\tilde{\phi}^{i}\right)^{\dagger}=\tilde{\phi}^{i}, \quad \text { i.e. } \quad\left(\phi^{i}\right)^{\dagger}=\Omega \phi^{i} \text {. }
$$

Then (4.4) reduces to the ordinary fuzzy sphere relation

$$
\left[\tilde{\phi}^{i}, \tilde{\phi}^{j}\right]=i \epsilon_{i j k} \tilde{\phi}^{k},
$$

generated by $\tilde{\phi}^{i}$, as well as to the relation $\tilde{\phi}^{i} \tilde{\phi}^{i}=R^{2}$. This justifies to call the noncommutative space generated by $\phi^{i}$ a twisted fuzzy sphere. It is remarkable that this construction is possible only for $\mathbb{Z}_{3}$ and for no other $\mathbb{Z}_{n}$, thus providing a justification for our choice of orbifold group.

An interesting realization of a twisted fuzzy sphere (4.4) is given by

$$
\phi^{i}=\Omega\left(\mathbb{1}_{3} \otimes \lambda_{(N)}^{i}\right)
$$

where $\lambda_{(N)}^{i}$ denote the generators of $S U(2)$ in the $N$-dimensional irreducible representation and the matrix $\Omega$ is given by

$$
\Omega=\Omega_{3} \otimes \mathbb{1}_{N}, \quad \Omega_{3}=\left(\begin{array}{ccc}
0 & 1 & 0 \\
0 & 0 & 1 \\
1 & 0 & 0
\end{array}\right), \quad \Omega^{3}=\mathbb{1} .
$$

The transformation $\phi^{i}=\Omega \tilde{\phi}^{i}$ (4.5) relates the "off-diagonal" orbifold sectors (4.2) to blockdiagonal configurations as follows,

$$
\phi^{i}=\left(\begin{array}{ccc}
0 & \left(\lambda_{(N)}^{i}\right)_{(N, \bar{N}, 1)} & 0 \\
0 & 0 & \left(\lambda_{(N)}^{i}\right)_{(1, N, \bar{N})} \\
\left(\lambda_{(N)}^{i}\right)_{(\bar{N}, 1, N)} & 0 & 0
\end{array}\right)=\Omega\left(\begin{array}{ccc}
\lambda_{(N)}^{i} & 0 & 0 \\
0 & \lambda_{(N)}^{i} & 0 \\
0 & 0 & \lambda_{(N)}^{i}
\end{array}\right) .
$$

We observe that the untwisted fields $\tilde{\phi}^{i}$, which generate the fuzzy sphere, acquire a blockdiagonal form. Each one of these blocks satisfies separately the fuzzy sphere relation (4.8) and therefore it is natural to reinterpret this configuration as three fuzzy spheres of fuzziness $N$. The solution $\phi^{i}$ can thus be interpreted as twisted configuration of three fuzzy spheres compatible with the orbifolding.

The solution (4.9) breaks completely the gauge symmetry $S U(N)^{3}$. This geometrical interpretation is helpful to understand the fluctuations around these fuzzy orbifolds. However, for our purposes it will be useful to consider solutions which do not break the $S U(N)^{3}$ gauge symmetry completely but they break it down to a smaller gauge group. We shall study such solutions in the following paragraph and present specific applications in the upcoming sections.

\footnotetext{
${ }^{12}$ Here $\left[\Omega, \phi^{i}\right]$ is understood before the orbifolding.
} 


\subsection{Dynamical generation of twisted fuzzy spheres}

Let us now show how the above geometries can arise as a vacuum solution of the field theory which was considered in Section 4.1. As it was previously described, the superpotential of the theory after the orbifold projection has the form (4.3). Therefore one can easily read off the corresponding potential, which is ${ }^{13}$

$$
V_{\mathcal{N}=1}^{(p r o j)}(\phi)=\frac{1}{4} \operatorname{Tr}\left(\left[\phi^{i}, \phi^{j}\right]^{\dagger}\left[\phi^{i}, \phi^{j}\right]\right)
$$

where $\phi^{i}$ denotes the scalar component of the superfield $\Phi^{i}$. The minimum of this potential is obtained for vanishing vevs of the fields and therefore vacua corresponding to non-commutative geometries do not exist without any additional modifications.

Clearly, in order to determine a minimum of the potential of the form (4.4) we have to make the following modifications in the theory. First of all, we have to add $\mathcal{N}=1$ soft supersymmetry breaking (SSB) terms of the form ${ }^{14}$

$$
V_{\mathrm{SSB}}=\frac{1}{2} \sum_{i} m_{i}^{2} \phi^{i \dagger} \phi^{i}+\frac{1}{2} \sum_{i, j, k} h_{i j k} \phi^{i} \phi^{j} \phi^{k}+\text { h.c. },
$$

where $h_{i j k}$ vanishes unless $i+j+k \equiv 0 \bmod 3$. Of course a set of SSB terms in the potential is necessary anyway in order for the theory to have a chance to become realistic, see e.g. [62]. After the addition of these soft terms as well as of the $D$-terms the full potential of the theory becomes

$$
V=V_{\mathcal{N}=1}^{(\text {proj })}+V_{\mathrm{SSB}}+V_{D}
$$

where $V_{D}=\frac{1}{2} D^{2}=\frac{1}{2} D^{I} D_{I}$ includes the $D$-terms of the theory. These $D$-terms have the form $D^{I}=\phi_{i}^{\dagger} T^{I} \phi^{i}$, where $T^{I}$ are the generators of the representation of the corresponding chiral multiplets.

In order to allow for twisted fuzzy sphere vacua, we now make the choice $h_{i j k}=\epsilon_{i j k}$ and $m_{i}^{2}=1$. A more general possibility will be investigated in Section 4.5. Then the potential (4.13) can be brought in the form

$$
V=\frac{1}{4}\left(F^{i j}\right)^{\dagger} F^{i j}+V_{D}
$$

where we have defined

$$
F^{i j}=\left[\phi^{i}, \phi^{j}\right]-i \epsilon^{i j k}\left(\phi^{k}\right)^{\dagger} .
$$

The first term of the potential is positive definite, and vanishes if the relation (4.4) holds. Therefore the global minimum of the potential is realized by a twisted fuzzy sphere $\tilde{S}_{N}^{2}(4.4)$, at least for a suitable range of parameters in the potential. The quartic term $V_{D}$ will typically only modify its radius, as in the case of the ordinary fuzzy sphere [20,31]. The expression (4.14) will be interpreted in the following as the field strength on the spontaneously generated fuzzy extra dimensions. Let us note that in general the potential may have several different local minima, which may be given e.g. by twisted fuzzy spheres with various radii; we will not discuss possible meta-stable vacua or phase-transitions here.

\footnotetext{
${ }^{13}$ Here we restrict to the scalar sector, since this is the relevant one for the search of fuzzy sphere vacua. Moreover, the gauge indices are suppressed.

${ }^{14}$ Here we present a set of scalar SSB terms. However, there exist of course other soft terms such as $\frac{1}{2} M \lambda \lambda$, where $\lambda$ is the gaugino and $M$ its mass, which has to be included in the full SSB sector [62].
} 
Let us now study further the vacuum and its geometric interpretation. The scalar fields $\phi^{i}$ are governed by the potential (4.13), which includes the F- and D-terms as well as the SSB terms. Under suitable conditions, this potential clearly has a twisted fuzzy sphere solution

$$
\phi^{i}=\Omega\left(\mathbb{1}_{3} \otimes\left(\lambda_{(N-n)}^{i} \oplus 0_{n}\right)\right)
$$

where $0_{n}$ denotes the $n \times n$ matrix with vanishing entries. The gauge symmetry is broken from $S U(N)^{3}$ down to $S U(n)^{3}$. This vacuum should be interpreted as $\mathbb{R}^{4} \times \tilde{S}_{N}^{2}$ with a twisted fuzzy sphere in the $\phi_{i}$ coordinates.

In order to understand the fluctuations of the scalar fields around this vacuum, the transformation $\phi^{i}=\Omega \tilde{\phi}^{i}$ is useful. Fluctuations around the ordinary fuzzy sphere $S_{N}^{2}$ are known to describe gauge and scalar fields on $S_{N}^{2}[27,31]$, and in particular they all become massive from the point of view of $\mathbb{R}^{4}$. We have seen in (4.11) that the twisted sphere $\tilde{S}_{N}^{2}$ is mapped by $\Omega$ into three fuzzy spheres $\tilde{\phi}^{i}$ embedded in the diagonal $N \times N$ blocks of the original $3 N \times 3 N$ matrix. Therefore all fluctuations can be understood as fields on the three diagonally embedded untwisted fuzzy spheres:

$$
\tilde{\phi}^{i}=\lambda_{(N)}^{i}+A^{i}
$$

and the field strength (4.14) reduces to the field strength on a fuzzy sphere

$$
F^{i j}=\left[\phi^{i}, \phi^{j}\right]-i \epsilon^{i j k}\left(\phi^{k}\right)^{\dagger}=\Omega^{2}\left(\left[\tilde{\phi}^{i}, \tilde{\phi}^{j}\right]-i \epsilon^{i j k} \tilde{\phi}^{k}\right)
$$

as long as (4.6) and (4.7) hold. The vacuum can thus be interpreted at intermediate energy scales as $\mathbb{R}^{4} \times S_{N}^{2}$ with three (untwisted) fuzzy spheres in the $\tilde{\phi}_{i}$ coordinates. Moreover, due to the orbifolding condition there are no off-diagonal components relating these different spheres. It now follows as in $[20,21]$ that the gauge fields and fermions can be decomposed into KaluzaKlein towers of massive modes on $S_{N}^{2}$ resp. $\tilde{S}_{N}^{2}$ due to the Higgs effect, as well as a massless sector.

\subsection{Chiral models from the fuzzy orbifold}

In this section we discuss three particular models which can be constructed in the above context. In all cases we start by considering the $\mathcal{N}=4$ SYM theory in four dimensions with gauge group $S U(3 N)$. As we have already mentioned this theory contains, in $\mathcal{N}=1$ language, an $S U(3 N)$ vector supermultiplet and three adjoint chiral supermultiplets $\Phi^{i}$ with superpotential

$$
W_{\mathcal{N}=4}=\epsilon_{i j k} \operatorname{Tr}\left(\Phi^{i} \Phi^{j} \Phi^{k}\right) .
$$

Subsequently we choose the discrete group $\mathbb{Z}_{3}$ and embed it in the $S U(3)$ part of the $R$ symmetry. Performing the orbifold projection, as it was described in Section 2, we obtain an

$\mathcal{N}=1$ theory with vectors in $S U(N)^{3}$ and complex scalars and fermions in chiral representations of the gauge group. In particular, according to (4.2), there are three families, each transforming under the gauge group $H$ as

$$
(N, \bar{N}, 1)+(\bar{N}, 1, N)+(1, N, \bar{N}) .
$$

Moreover, the superpotential takes the form (4.3). The difference between the models lies in the next step of the construction, where the gauge group $S U(N)^{3}$ will be broken spontaneously to a unification group. The minimal cases which satisfy the requirement of anomaly freedom are the gauge groups $S U(4) \times S U(2) \times S U(2), S U(4)^{3}$ and $S U(3)^{3}$. 


\subsubsection{A $S U(4)_{c} \times S U(2)_{L} \times S U(2)_{R}$ model}

In order to obtain the Pati-Salam gauge group $S U(4)_{c} \times S U(2)_{L} \times S U(2)_{R}$ [63] (see also [64] and [65] for a recent study), we decompose the integer $N$ in two different ways, namely as

$$
N=n_{1}+4, \quad N=n_{2}+2 .
$$

Then we consider the following regular embeddings,

$$
S U(N) \supset S U\left(n_{1}\right) \times S U(4) \times U(1), \quad S U(N) \supset S U\left(n_{2}\right) \times S U(2) \times U(1) .
$$

The full gauge group is accordingly decomposed as

$$
S U(N)^{3} \supset S U\left(n_{1}\right) \times S U(4) \times S U\left(n_{2}\right) \times S U(2) \times S U\left(n_{2}\right) \times S U(2) \times U(1)^{3} .
$$

Performing a shuffling of the group factors and ignoring the $U(1) \mathrm{s}^{15}$ it is easy to see that the original representations (4.2) are decomposed as follows,

$$
\begin{aligned}
& S U\left(n_{1}\right) \times S U\left(n_{2}\right) \times S U\left(n_{2}\right) \times S U(4) \times S U(2) \times S U(2), \\
& \left(n_{1}, \bar{n}_{2}, 1 ; 1,1,1\right)+\left(1, n_{2}, \bar{n}_{2} ; 1,1,1\right)+\left(\bar{n}_{1}, 1, n_{2} ; 1,1,1\right)+(1,1,1 ; 4,2,1)+(1,1,1 ; 1,2,2) \\
& \quad+(1,1,1 ; \overline{4}, 1,2)+\left(n_{1}, 1,1 ; 1,2,1\right)+\left(1, n_{2}, 1 ; 1,1,2\right)+\left(1,1, n_{2} ; \overline{4}, 1,1\right) \\
& \quad+\left(\bar{n}_{1}, 1,1 ; 1,1,2\right)+\left(1, \bar{n}_{2}, 1 ; 4,1,1\right)+\left(1,1, \bar{n}_{2} ; 1,2,1\right) .
\end{aligned}
$$

First of all it is important to note that the theory is anomaly free. This is merely due to the special feature of $S U(2)$, where the fundamental representation is self-conjugate. Therefore, although the structure involves a product of different gauge groups, it is still not anomalous.

Now utilizing the mechanism of Section 3, fuzzy extra dimensions can be dynamically generated and the unbroken gauge group at low-energies is $S U(4)_{c} \times S U(2)_{L} \times S U(2)_{R}$, with fields transforming under the representations

$$
\begin{aligned}
& S U(4) \times S U(2) \times S U(2), \\
& 3 \cdot((4,2,1)+(\overline{4}, 1,2)+(1,2,2)) .
\end{aligned}
$$

This is realized by the following vacuum

$$
\phi^{i}=\Omega\left(0_{2} \oplus \mathbb{1}_{3} \otimes\left(\lambda_{(N-2)}^{i} \oplus 0_{2}\right)\right), \quad \Omega=\left(\begin{array}{cc}
\mathbb{1}_{2} & 0 \\
0 & \Omega_{3} \otimes \mathbb{1}_{N}
\end{array}\right)
$$

interpreted in terms of twisted fuzzy spheres, where $\Omega_{3}$ is defined in (4.10).

Then the quarks and leptons of the SM fit in these representations. For example, the first generation is represented as

$$
f \sim(4,2,1)=\left(\begin{array}{cc}
d_{L}^{1} & u_{L}^{1} \\
d_{L}^{2} & u_{L}^{2} \\
d_{L}^{3} & u_{L}^{3} \\
e_{L} & \nu_{L}
\end{array}\right), \quad f^{c} \sim(\overline{4}, 1,2)=\left(\begin{array}{cccc}
d_{L}^{1 c} & d_{L}^{2 c} & d_{L}^{3 c} & e_{L}^{c} \\
u_{L}^{1 c} & u_{L}^{2 c} & u_{L}^{3 c} & \nu_{L}^{c}
\end{array}\right)
$$

and similarly for the other two generations. Moreover, the $h \sim(1,2,2)$ representation involves the Higgses and the Higgsini.

\footnotetext{
${ }^{15}$ These may be anomalous and become massive by the Green-Schwarz mechanism and therefore they decouple at low energies [60].
} 


\subsubsection{A $S U(4)_{c} \times S U(4)_{L} \times S U(4)_{R}$ model}

A further possibility is the gauge group $S U(4)_{c} \times S U(4)_{L} \times S U(4)_{R}$, where $S U(4)_{c}$ is again the Pati-Salam colour gauge group. This gauge group can be obtained by decomposing $N$ as

$$
N=n+4
$$

leading to the decomposition of $S U(N)^{3}$ to $S U(n)^{3} \times S U(4)^{3}$ with particle content

$$
\begin{aligned}
& S U(n) \times S U(n) \times S U(n) \times S U(4) \times S U(4) \times S U(4) \\
& \quad(n, \bar{n}, 1 ; 1,1,1)+(1, n, \bar{n} ; 1,1,1)+(\bar{n}, 1, n ; 1,1,1)+(1,1,1 ; 4, \overline{4}, 1) \\
& \quad+(1,1,1 ; 1,4, \overline{4})+(1,1,1 ; \overline{4}, 1,4)+(n, 1,1 ; 1, \overline{4}, 1)+(1, n, 1 ; 1,1, \overline{4}) \\
& \quad+(1,1, n ; \overline{4}, 1,1)+(\bar{n}, 1,1 ; 1,1,4)+(1, \bar{n}, 1 ; 4,1,1)+(1,1, \bar{n} ; 1,4,1) .
\end{aligned}
$$

This is realized by the following vacuum, interpreted in terms of twisted fuzzy spheres $\tilde{S}_{N-4}^{2}$ as in (4.15):

$$
\phi^{i}=\Omega\left(\mathbb{1}_{3} \otimes\left(\lambda_{(N-4)}^{i} \oplus 0_{4}\right)\right)
$$

where $\Omega$ is defined in (4.10). Decomposing $S U(N) \supset S U(n) \times S U(4) \times U(1)$, the gauge group is broken to $S U(4)^{3}$, and the low-energy field content is

$$
\begin{aligned}
& S U(4) \times S U(4) \times S U(4), \\
& 3 \cdot((4, \overline{4}, 1)+(\overline{4}, 1,4)+(1,4, \overline{4})) .
\end{aligned}
$$

This case has been examined originally in [66] and from a phenomenological viewpoint in [67]. The quarks and leptons of the first family should transform as

$$
f=\left(\begin{array}{llll}
d & u & y & x \\
d & u & y & x \\
d & u & y & x \\
e & \nu & a & v
\end{array}\right) \sim(4, \overline{4}, 1), \quad f^{c}=\left(\begin{array}{llll}
d^{c} & d^{c} & d^{c} & e^{c} \\
u^{c} & u^{c} & u^{c} & \nu^{c} \\
y^{c} & y^{c} & y^{c} & a^{c} \\
x^{c} & x^{c} & x^{c} & v^{c}
\end{array}\right) \sim(\overline{4}, 1,4)
$$

Clearly, there have to be new heavy quarks and leptons and in addition the supermultiplet $h \sim(1,4, \overline{4})$ still has to be considered.

A very interesting feature which we would like to point out here is that the one-loop $\beta$-function coefficient in the renormalization group equation of each $S U(4)$ gauge coupling is given by

$$
b=\left(-\frac{11}{3}+\frac{2}{3}\right) \cdot 3+n_{f}\left(\frac{2}{3}+\frac{1}{3}\right) \cdot \frac{1}{2} \cdot 2 \cdot 3
$$

which for the present case of $n_{f}=3$ copies of the supermultiplet (4.17) results in

$$
b=0 .
$$

Therefore, we observe that the existence of three families of quarks and leptons leads to one of the necessary conditions for a finite field theory. Let us mention that this is a general feature of models with a $S U(N)^{k}$ gauge group, independently of the values of $N$ and $k$ [67]. Therefore it also holds in the following case of $S U(3)^{3}$. 


\subsubsection{A $S U(3)_{c} \times S U(3)_{L} \times S U(3)_{R}$ model}

Let us now turn to another possibility, the trinification group $S U(3)_{c} \times S U(3)_{L} \times S U(3)_{R}[68,69]$, which was also studied in $[67,70,71,72,73]$ and from a string theory perspective in [74]. In the present case we consider the following picture. Let us decompose the integer $N$ as

$$
N=n+3
$$

Subsequently, let us consider the regular embedding

$$
S U(N) \supset S U(n) \times S U(3) \times U(1) .
$$

Then the relevant embedding for the full gauge group is

$$
S U(N)^{3} \supset S U(n) \times S U(3) \times S U(n) \times S U(3) \times S U(n) \times S U(3) \times U(1)^{3} .
$$

The three $U(1)$ factors decouple from the low-energy sector of the theory, as it was mentioned above. The representations (4.16) are then decomposed accordingly (notice the shuffling in the group factors),

$$
\begin{aligned}
& S U(n) \times S U(n) \times S U(n) \times S U(3) \times S U(3) \times S U(3), \\
& \quad(n, \bar{n}, 1 ; 1,1,1)+(1, n, \bar{n} ; 1,1,1)+(\bar{n}, 1, n ; 1,1,1)+(1,1,1 ; 3, \overline{3}, 1) \\
& \quad+(1,1,1 ; 1,3, \overline{3})+(1,1,1 ; \overline{3}, 1,3)+(n, 1,1 ; 1, \overline{3}, 1)+(1, n, 1 ; 1,1, \overline{3}) \\
& \quad+(1,1, n ; \overline{3}, 1,1)+(\bar{n}, 1,1 ; 1,1,3)+(1, \bar{n}, 1 ; 3,1,1)+(1,1, \bar{n} ; 1,3,1) .
\end{aligned}
$$

This is realized by the following vacuum, interpreted in terms of twisted fuzzy spheres $\tilde{S}_{N-3}^{2}$ as in (4.15):

$$
\phi^{i}=\Omega\left[\mathbb{1}_{3} \otimes\left(\lambda_{(N-3)}^{i} \oplus 0_{3}\right)\right] .
$$

Considering the decomposition (4.18), the gauge group is broken to $K=S U(3)^{3}$. Finally, the surviving fields under the unbroken gauge group $K$ transform in the following representations,

$$
\begin{aligned}
& S U(3) \times S U(3) \times S U(3), \\
& 3 \cdot((3, \overline{3}, 1)+(\overline{3}, 1,3)+(1,3, \overline{3})) .
\end{aligned}
$$

These are the desired chiral representations of the unification group $S U(3)_{c} \times S U(3)_{L} \times S U(3)_{R}$. The quarks of the first family transform under the gauge group as

$$
q=\left(\begin{array}{ccc}
d & u & h \\
d & u & h \\
d & u & h
\end{array}\right) \sim(3, \overline{3}, 1), \quad q^{c}=\left(\begin{array}{lll}
d^{c} & d^{c} & d^{c} \\
u^{c} & u^{c} & u^{c} \\
h^{c} & h^{c} & h^{c}
\end{array}\right) \sim(\overline{3}, 1,3),
$$

and the leptons transform as

$$
\lambda=\left(\begin{array}{ccc}
N & E^{c} & \nu \\
E & N^{c} & e \\
\nu^{c} & e^{c} & S
\end{array}\right) \sim(1,3, \overline{3})
$$

Similarly, the corresponding matrices for the quarks and leptons of the other two families can be written down. 


\subsubsection{A closer look at the masses}

A vital issue of our construction is whether there exist massless and massive modes at the same time. Clearly we need both of these sets; the massless modes in order to obtain chiral fermions and the massive modes in order to reproduce the Kaluza-Klein tower and provide undoubtful justification that the theory develops fuzzy extra dimensions.

A way to see this through the embeddings we presented before is the following. Let us work out the case of $S U(3)^{3}$, since the same arguments apply to the other two cases as well. Under the final gauge group $S U(3)^{3}$ the fermions transform in the representations (4.21), hence they are chiral. Therefore they remain massless since they are protected by chiral symmetry.

On the other hand, looking at (4.19) we can make two crucial observations. First of all, it becomes clear from the vacuum solution (4.20) that the scalar fields which acquire vevs in this vacuum are the following,

$$
\langle(n, \bar{n}, 1 ; 1,1,1)\rangle, \quad\langle(1, n, \bar{n} ; 1,1,1)\rangle, \quad\langle(\bar{n}, 1, n ; 1,1,1)\rangle .
$$

Then all the fermions, apart from the chirally protected ones, obtain masses, since we can form the invariants

$$
\begin{aligned}
& (1, n, \bar{n} ; 1,1,1)\langle(n, \bar{n}, 1 ; 1,1,1)\rangle(\bar{n}, 1, n ; 1,1,1)+\text { cyclic permutations, } \\
& (\bar{n}, 1,1 ; 1,1,3)\langle(n, \bar{n}, 1 ; 1,1,1)\rangle(1, n, 1 ; 1,1, \overline{3}) \quad \text { etc. }
\end{aligned}
$$

and the corresponding ones for all the other fermions. In these invariants the field in the middle is the scalar field which acquires the vev (4.20), while the other two are fermions, i.e. the invariants are trilinear Yukawa terms and they are responsible for the fermion masses after the spontaneous symmetry breaking. Therefore a finite Kaluza-Klein tower of massive fermionic modes appears, consistent with the interpretation of the vacuum (4.20) as a higher-dimensional theory with spontaneously generated fuzzy extra dimensions. In particular, the fluctuations from this vacuum correspond to the internal components of the higher-dimensional gauge field. Also, as far as the fermions transforming as $(1,1,1 ; 3, \overline{3}, 1),(1,1,1 ; \overline{3}, 1,3)$ and $(1,1,1 ; 1,3, \overline{3})$ are concerned, obviously there does not exist any trilinear invariant that they could form with one of the scalar fields which acquire a vev. Therefore, as it was already mentioned, they remain massless and they are the chiral fermions of the model.

Finally, it is worth noting that in (4.24) the "internal" structure and the "observable", lowenergy structure appear mixed and therefore these Kaluza-Klein fermion masses may have an effect on the $S U(3)^{3}$ phenomenology [75, 76, 77, 78].

\subsection{Fuzzy breaking for $S U(3)^{3}$}

In this section we discuss another possible application of the fuzzy orbifold construction which was presented above. We focus on the most interesting case ${ }^{16}$ of $S U(3)^{3}$ and the orbifold projection is utilized to study its spontaneous breaking down to the MSSM and the $S U(3)_{c} \times$ $U(1)_{\mathrm{em}}$. It is important to note that we shall focus only on symmetry breaking patterns where additional superfields are not introduced, namely the model is broken spontaneously due to its own scalar sector.

In particular, instead of starting with a $S U(3 N)$ gauge theory with a large $N$, we can start with a smaller gauge group, namely $S U(9)$, in order to obtain the model $S U(3)^{3}$ after orbifolding. Therefore the initial set-up consists of the $\mathcal{N}=4 \mathrm{SYM}$ theory with gauge fields in the gauge group $S U(9)$. Subsequently a $\mathbb{Z}_{3}$ orbifold projection is performed in the spirit of Section 4.1.

\footnotetext{
${ }^{16}$ The breaking of the models with gauge group $S U(4) \times S U(2) \times S U(2)$ and $S U(4)^{3}$ was studied along the same lines in [23], where it was shown that they meet serious phenomenological obstacles.
} 
Alternatively, this procedure may be viewed as a second step of the construction which was presented in Section 4.4.3. Indeed, if such a view is adopted, after the large- $N$ symmetry breaking, the $S U(3)^{3}$ model is obtained. It involves a superpotential and the corresponding soft supersymmetry breaking terms. Part of the SSB sector is naturally inherited from the corresponding one in the large- $N$ model, namely it is already contained in the expression (4.12) for suitable $h_{i j k}$. This fact justifies further the use of the same technique, the spontaneous generation of twisted fuzzy spheres, in order to achieve the spontaneous symmetry breaking down to the MSSM and subsequently to the $S U(3) \times U(1)_{\mathrm{em}}$.

Specifically, the model is obtained from an $S U(9)$ gauge theory as follows. We perform an orbifold projection of the $\mathcal{N}=4, S U(9)$ SYM theory such that the $\gamma_{3}$ of equation (4.1) takes the form

$$
\gamma_{3}=\left(\begin{array}{ccc}
\mathbb{1}_{3} & 0 & 0 \\
0 & \omega \mathbb{1}_{3} & 0 \\
0 & 0 & \omega^{2} \mathbb{1}_{3}
\end{array}\right) .
$$

Then, according to the rules of Section 2, the gauge group breaks down to $S U(3)^{3}$, with three chiral supermultiplets transforming as

$$
\begin{aligned}
& S U(3) \times S U(3) \times S U(3), \\
& 3 \cdot((3, \overline{3}, 1)+(\overline{3}, 1,3)+(1,3, \overline{3})) .
\end{aligned}
$$

First of all, the quarks of the first family transform under the gauge group as in equation (4.22) and the leptons transform as in equation (4.23). The superpotential (4.3) after the orbifold projection in this case becomes [67]

$$
W_{\mathcal{N}=1}^{(\text {proj })}\left(\lambda, q, q^{c}\right)=Y \operatorname{Tr}\left(\lambda q^{c} q\right)+Y^{\prime} \epsilon_{i j k} \epsilon_{a b c}\left(\lambda_{i a} \lambda_{j b} \lambda_{k c}+q_{i a}^{c} q_{j b}^{c} q_{k c}^{c}+q_{i a} q_{j b} q_{k c}\right),
$$

where the family superscripts are suppressed. The last terms are special in the $S U(3)^{3}$ case, and may involve different families. The soft supersymmetry breaking terms, which are necessary in order to obtain vacua in the form of twisted fuzzy spheres, are correspondingly read off from equation (4.12) with the appropriate $h_{i j k}$ in order to incorporate different scales for the GUT and the EW symmetry breaking ${ }^{17}$.

The spontaneous breaking of this unification model down to the MSSM has been studied in several publications $[67,70,72]$ and it can be achieved in different ways. Here we would like to mention that in all the known symmetry breaking patterns either additional superfields have to be introduced in the theory [70] or the breaking has to happen in more than one steps, e.g. through the left-right symmetric model $S U(3) \times S U(2)_{L} \times S U(2)_{R} \times U(1)_{L+R}[67]$.

Here we would like to present a different symmetry breaking pattern, where the initial $S U(3)^{3}$ gauge symmetry is spontaneously broken due to the existing scalar sector of the model, i.e. without the need of any additional superfields, and moreover the breaking happens in one step. In order to achieve this we shall utilize the fuzzy orbifold techniques which were presented previously.

Let us recall that the fields of one family can be represented by the following matrix,

$$
\left(\begin{array}{ccc}
0_{3} & q & 0_{3} \\
0_{3} & 0_{3} & \lambda \\
q^{c} & 0_{3} & 0_{3}
\end{array}\right),
$$

where $0_{3}$ is the $3 \times 3$ matrix with all the entries zero. Obviously the quark blocks cannot acquire a vev, since this would break the colour $S U(3)$ gauge group factor. Therefore the term

\footnotetext{
${ }^{17}$ Of course the EW symmetry breaking of the MSSM requires the introduction of extra soft supersymmetry breaking terms (see e.g. [62]).
} 
$\operatorname{Tr}\left(\lambda q q^{c}\right)$ in the superpotential cannot play any role here. The block which corresponds to the lepton supermultiplet may acquire vevs only in the directions which have zero hypercharge. This means that out of the nine components of this block only five may acquire a vev, namely $S$, $\nu, \nu^{c}, N$ and $N^{c}$. The first three are responsible for the breaking down to the MSSM, while the last two take care of the EW breaking. Such a vacuum may indeed arise here due to the presence of the $\epsilon_{i j k} \epsilon_{a b c} \lambda_{i a} \lambda_{j b} \lambda_{k c}$ term in the superpotential, and moreover we can interpret it again in terms of a twisted fuzzy sphere.

To see the relation with a twisted fuzzy sphere (4.4), we transform the lepton matrices as $\lambda^{\prime i}=\Omega_{3} \lambda^{i}$ where $\Omega_{3}=\left(\begin{array}{lll}0 & 1 & 0 \\ 0 & 0 & 1 \\ 1 & 0 & 0\end{array}\right)$, noting that the relevant term $\epsilon_{i j k} \epsilon_{a b c} \lambda_{i a} \lambda_{j b} \lambda_{k c}$ is invariant (up to sign) under such a transformation. Then $\lambda$ is transformed to

$$
\lambda^{\prime}=\left(\begin{array}{ccc}
E & N^{c} & e \\
\nu^{c} & e^{c} & S \\
N & E^{c} & \nu
\end{array}\right)
$$

Now consider a vacuum solution of the form (superscripts here denote families):

$$
\lambda^{\prime 1}=\left(\begin{array}{ccc}
0 & k_{1} & 0 \\
0 & 0 & 0 \\
0 & 0 & 0
\end{array}\right), \quad \lambda^{\prime 2}=\left(\begin{array}{ccc}
0 & 0 & 0 \\
0 & 0 & k_{2} \\
0 & 0 & 0
\end{array}\right), \quad \lambda^{\prime 3}=\left(\begin{array}{ccc}
0 & 0 & 0 \\
0 & 0 & 0 \\
k_{3} & 0 & 0
\end{array}\right),
$$

while everything else acquires a vanishing vev. These vevs correspond to the directions of $N, N^{c}$ and $S$. The above matrices satisfy

$$
\left[\lambda^{\prime i}, \lambda^{\prime j}\right]=i h_{i j k}\left(\lambda^{\prime k}\right)^{\dagger}
$$

where we have defined again

$$
h_{i j k} \equiv \frac{k_{i} k_{j}}{k_{k}} \epsilon_{i j k} .
$$

This is a generalization of the twisted fuzzy sphere vacuum where more than one scales may be included. In the present model this is desirable, since at least two scales have to be introduced, corresponding to the GUT and EW breaking respectively. Moreover, since the model enjoys $\mathcal{N}=1$ supersymmetry these scales may in principle remain separate.

On the other hand, if we transform the lepton matrices as

$$
\lambda^{\prime \prime i}=\Omega_{3}^{\prime} \lambda^{i}, \quad \text { where } \quad \Omega_{3}^{\prime}=\left(\begin{array}{ccc}
0 & 1 & 0 \\
1 & 0 & 0 \\
0 & 0 & 1
\end{array}\right), \quad \text { then } \quad \lambda^{\prime \prime}=\left(\begin{array}{ccc}
E & N^{c} & e \\
N & E^{c} & \nu \\
\nu^{c} & e^{c} & S
\end{array}\right) .
$$

The same twisted fuzzy sphere vacuum as before, namely the matrices (4.25), corresponds now to the directions $\nu, \nu^{c}$ and $N^{c}$. Therefore, with the above procedure all the neutral directions acquire a vev and the original $S U(3)^{3}$ model is spontaneously broken down to $S U(3)_{c} \times U(1)_{\mathrm{em}}$. In particular, at the scale where the directions $\nu, \nu^{c}$ and $S$ acquire vevs, $S U(3)^{3}$ is spontaneously broken down to the MSSM. Subsequently, at the scale where the $N$ and $N^{c}$ directions acquire vevs the breaking down to $S U(3)_{c} \times U(1)_{\mathrm{em}}$ takes place. As we have already mentioned these scales are hopefully kept separate by supersymmetry. In other words the hierarchy problem is the same as in any supersymmetric particle physics model.

The remarkable new result of the above procedure is that the spontaneous breaking of the $S U(3)^{3}$ model acquires an interesting geometrical explanation. It takes place solely due to the Higgsing of the twisted fuzzy spheres in the extra dimensions, without the need of any additional superfields and without the need of any intermediate breaking. 


\section{Discussion and conclusions}

Non-commutative geometry has been regarded as a promising framework for constructing finite quantum field theories or at least as a natural scheme for regularizing quantum field theories. However the quantization of field theories on non-commutative spaces has turned out to be much more difficult than expected and with problematic ultraviolet features [79, 80], see however [81], and $[31,82]$.

Although SM type of models have been constructed using the Seiberg-Witten map, they can only be considered as effective theories and they are not renormalizable. A drastic change in the perspective of non-commutative geometry was given with the suggestion that indeed it might be relevant for particle physics models but in the description of extra dimensions [19]. The higher-dimensional theories that can be constructed based on this proposal, reviewed in the present article, appeared to have many interesting unexpected features ranging from their renormalizability to their predictivity.

In the first part of the review we have considered ideas from non-commutative geometry in order to construct particle physics models which could turn out to be phenomenologically viable. Specifically, in the context of higher-dimensional gauge theories, we explored the possibility that the extra dimensions are described by matrix approximations to smooth manifolds known as fuzzy spaces. Let us now summarize our results and discuss prospects of further work on this subject.

In the first part of this paper we considered higher-dimensional gauge theories defined on the product of Minkowski space and a fuzzy coset space $(S / R)_{F}$ and their dimensional reduction to four dimensions using the CSDR scheme. Although for the technicalities one has to consult the detailed exposition in Section 2, a major difference between fuzzy and ordinary CSDR is that in the fuzzy case one always embeds $S$ in the gauge group $G$ instead of embedding just $R$ in $G$. This is due to the fact that the differential calculus on the fuzzy coset space is based on $\operatorname{dim} S$ derivations instead of the restricted $\operatorname{dim} S-\operatorname{dim} R$ used in the ordinary one. As a result the four-dimensional gauge group $H=C_{G}(R)$ appearing in the ordinary CSDR after the geometrical breaking and before the spontaneous symmetry breaking due to the four-dimensional Higgs fields does not appear in the fuzzy CSDR. In fuzzy CSDR the spontaneous symmetry breaking mechanism takes already place by solving the fuzzy CSDR constraints. The four-dimensional potential has the typical "mexican hat" shape, but it appears already shifted to a minimum. Therefore in four dimensions appears only the physical Higgs field that survives after a spontaneous symmetry breaking. Correspondingly in the Yukawa sector of the theory we have the results of the spontaneous symmetry breaking, i.e. massive fermions and Yukawa interactions among fermions and the physical Higgs field. Having massive fermions in the final theory is a generic feature of CSDR when $S$ is embedded in $G$ [4]. We see that if one would like to describe the spontaneous symmetry breaking of the SM in the present framework, then one would be naturally led to large extra dimensions.

A fundamental difference between the ordinary CSDR and its fuzzy version is the fact that a non-Abelian gauge group $G$ is not really required in high dimensions. Indeed the presence of a $U(1)$ in the higher-dimensional theory is enough to obtain non-Abelian gauge theories in four dimensions.

In a further development, we have presented a renormalizable four-dimensional $S U(N)$ gauge theory with a suitable multiplet of scalars, which dynamically develops fuzzy extra dimensions that form a fuzzy sphere. The model can then be interpreted as 6-dimensional gauge theory, with gauge group and geometry depending on the parameters in the original Lagrangian. We explicitly calculate the tower of massive Kaluza-Klein modes, consistent with an interpretation as compactified higher-dimensional gauge theory, and determine the effective compactified gauge 
theory. This model has a unique vacuum, with associated geometry and low-energy gauge group depending only on the parameters of the potential.

There are many remarkable aspects of this model. First, it provides an extremely simple and geometrical mechanism of dynamically generating extra dimensions, since it is based on a basic lesson from non-commutative gauge theory, namely that non-commutative or fuzzy spaces can be obtained as solutions of matrix models. The mechanism is quite generic, and does not require fine-tuning or supersymmetry. This provides in particular a realization of the basic ideas of compactification and dimensional reduction within the framework of renormalizable quantum field theory. Moreover, we are essentially considering a large $N$ gauge theory, which should allow to apply the analytical techniques developed in this context.

In particular, it turns out that the generic low-energy gauge group is given by $S U\left(n_{1}\right) \times$ $S U\left(n_{2}\right) \times U(1)$ or $S U(n)$, while gauge groups which are products of more than two simple components (apart from $U(1)$ ) do not seem to occur in this model. The values of $n_{1}$ and $n_{2}$ are determined dynamically. Moreover, a magnetic flux is induced in the vacua with non-simple gauge group.

The inclusion of fermions in the above class of models showed that the best one could achieve so far is to obtain theories with mirror fermions in bifundamental representations of the lowenergy gauge group [21, 22]. Indeed, studying in detail the fermionic sector of a model which dynamically develops extra dimensions with the geometry of fuzzy $S^{2} \times S^{2}$ we found out that the low-energy theory contains two mirror sectors, even when magnetic fluxes are included on the two fuzzy spheres. Although mirror fermions do not exclude the possibility to make contact with phenomenology [48], it would be desirable to obtain exactly chiral fermions.

In order to pursue further the possibility to obtain chiral fermions we introduced an additional structure in the above context, based on orbifolds, in order to obtain chiral low-energy models. In particular we performed a $\mathbb{Z}_{3}$ orbifold projection of a $\mathcal{N}=4 S U(3 N)$ SYM theory, which leads to a $\mathcal{N}=1$ supersymmetric theory with gauge group $S U(N)^{3}$. Adding a suitable set of soft supersymmetry breaking terms in the $\mathcal{N}=1$ theory, certain vacua of the theory were revealed, where twisted fuzzy spheres are dynamically generated. It is well known that the introduction of a soft supersymmetry breaking sector is not only natural but also necessary in the constructions of phenomenologically viable supersymmetric theories, with prime example the case of the MSSM [62]. Such vacua correspond to models which behave at intermediate energy scales as higher-dimensional theories with a finite Kaluza-Klein tower of massive modes and a chiral low-energy spectrum. The most interesting chiral models for low-energy phenomenology which can be constructed in this context turn out to be $S U(4) \times S U(2) \times S U(2), S U(4)^{3}$ and $S U(3)^{3}$.

Subsequently, the possibility to achieve further breaking of the above models down to the MSSM and $S U(3)_{c} \times U(1)_{\mathrm{em}}$ using twisted fuzzy spheres was studied and it was shown that this is indeed possible. Thus the spontaneous symmetry breaking of these unification groups acquires an interesting geometrical explanation in terms of twisted fuzzy spheres. The most interesting case is the trinification group $S U(3)^{3}$, which can be promoted even to an all-loop finite theory (for a review see [83]) and therefore it is suitable to make predictions [67, 71].

We have thus shown that fuzzy extra dimensions can arise in simple field-theoretical models which are chiral, renormalizable, and may be phenomenologically viable. Moreover, since some of these models can be finite with fermions in the adjoint of an underlying $S U(3 N)$ gauge group, these models can be generalized into the framework of Yang-Mills matrix model such as [84, 85].

We have already argued about the importance of the soft supersymmetry breaking terms in the above models. It would be very interesting to explore further the possibility to obtain these terms directly from a higher-dimensional gauge theory. In fact it is known that the SSB terms arise naturally in four dimensions when the ordinary CSDR scheme is used [86, 87]. Therefore it is natural to expect that applying the fuzzy CSDR scheme to an orbifolded higher-dimensional 
gauge theory coupled to fermions one could naturally obtain the models we discussed above without having to add the SSB terms by hand. We plan to report on this soon.

\section{A Clifford algebra conventions}

In this appendix we collect our conventions on the representations of the Clifford algebras we have used in Section 3.

The gamma matrices $\gamma_{\mu}$ define the four-dimensional Clifford algebra and they are chosen to be purely imaginary, corresponding to the Majorana representation in four dimensions. In our conventions this representation is explicitly given by the matrices

$$
\gamma_{0}=\sigma_{0} \otimes \sigma_{2}, \quad \gamma_{1}=i \sigma_{0} \otimes \sigma_{3}, \quad \gamma_{2}=i \sigma_{1} \otimes \sigma_{1}, \quad \gamma_{3}=i \sigma_{3} \otimes \sigma_{1},
$$

where $\sigma_{0}:=\mathbb{1}_{2}$ is the identity matrix.

Moreover, we give here the explicit Majorana representation of the six-dimensional Clifford algebra, which is known to exist in six Euclidean dimensions. This is naturally adapted to $S O(3)_{L} \times S O(3)_{R} \subset S O(6)$, and closely related to other constructions, see for example [47]. First we consider the matrices

$$
\begin{array}{lll}
\gamma_{L}^{1}=\sigma^{1} \otimes \sigma^{2}, & \gamma_{L}^{2}=\sigma^{2} \otimes \mathbb{1}, & \gamma_{L}^{3}=\sigma^{3} \otimes \sigma^{2}, \\
\gamma_{R}^{1}=\sigma^{2} \otimes \sigma^{1}, & \gamma_{R}^{2}=-\sigma^{2} \otimes \sigma^{3}, & \gamma_{R}^{3}=\mathbb{1} \otimes \sigma^{2}, *
\end{array}
$$

which are antisymmetric and purely imaginary, hence hermitian, and they satisfy

$$
\gamma_{L}^{i} \gamma_{L}^{j}=\delta^{i j}+i \epsilon_{k}^{i j} \gamma_{L}^{k}, \quad \gamma_{R}^{i} \gamma_{R}^{j}=\delta^{i j}+i \epsilon_{k}^{i j} \gamma_{R}^{k}, \quad\left[\gamma_{L}^{i}, \gamma_{R}^{j}\right]=0
$$

Then the following matrices define a representation of the $S O(6)$ Clifford algebra

$$
\Delta_{i}=i \sigma_{1} \otimes \gamma_{L}^{i}, \quad \Delta_{3+i}=i \sigma_{3} \otimes \gamma_{R}^{i},
$$

satisfying the desired relation

$$
\left\{\Delta^{\mu}, \Delta^{\nu}\right\}=-2 \delta^{\mu \nu}
$$

They are manifestly anti-symmetric and real, hence they furnish a Majorana representation. The left and right chiral projections are given by

$$
\Gamma_{L}^{(Y)}=\Delta_{1} \Delta_{2} \Delta_{3}=\sigma_{1} \otimes \mathbb{1}, \quad \Gamma_{R}^{(Y)}=\Delta_{4} \Delta_{5} \Delta_{6}=\sigma_{3} \otimes \mathbb{1}
$$

and the six-dimensional chirality operator is

$$
\Gamma^{(Y)}=-i \Gamma_{L}^{(Y)} \Gamma_{R}^{(Y)}=-\sigma_{2} \otimes \mathbb{1} .
$$

\section{Acknowledgements}

The authors would like to thank P. Aschieri, T. Grammatikopoulos, J. Madore, P. Manousselis and $\mathrm{H}$. Steinacker for their collaboration on the subjects of this report over the last years. This work is supported by the NTUA programmes for basic research PEVE 2008 and 2009, the European Union's RTN programme under contract MRTN-CT-2006-035505 and the European Union's ITN programme "UNILHC" PITN-GA-2009-237920. 


\section{References}

[1] Green M.B., Schwarz J.H., Witten E., Superstring theory. Vol. 1. Introduction, Cambridge Monographs on Mathematical Physics, Cambridge University Press, Cambridge, 1987.

Green M.B., Schwarz J.H., Witten E., Superstring theory. Vol. 2. Loop amplitudes, anomalies and phenomenology, Cambridge Monographs on Mathematical Physics, Cambridge University Press, Cambridge, 1987.

Polchinski J., String theory. Vol. 1. An introduction to the bosonic string, Cambridge University Press, Cambridge, 1998.

Polchinski J., String theory. Vol. 2. Superstring theory and beyond, Cambridge University Press, Cambridge, 1998.

Lüst D., Theisen S., Lectures on string theory, Lecture Notes in Physics, Vol. 346, Springer-Verlag, Berlin, 1989.

[2] Gross D.J., Harvey J.A., Martinec E.J., Rohm R., Heterotic string theory. I. The free heterotic string, Nuclear Phys. B 256 (1985), 253-284.

[3] Forgács P., Manton N.S., Space-time symmetries in gauge theories, Comm. Math. Phys. 72 (1980), 15-35.

[4] Kapetanakis D., Zoupanos G., Coset-space-dimensional reduction of gauge theories, Phys. Rep. 219 (1992), 76 pages.

[5] Kubyshin Yu.A., Mourão J.M., Rudolph G., Volobujev I.P., Dimensional reduction of gauge theories, spontaneous compactification and model building, Lecture Notes in Physics, Vol. 349, Springer-Verlag, Berlin, 1989.

[6] Scherk J., Schwarz J.H., How to get masses from extra dimensions, Nuclear Phys. B 153 (1979), 61-88.

[7] Manton N.S., Fermions and parity violation in dimensional reduction schemes, Nuclear Phys. B 193 (1981), $502-516$.

Chapline G., Slansky R., Dimensional reduction and flavor chirality, Nuclear Phys. B 209 (1982), 461-483.

[8] Connes A., Noncommutative geometry, Academic Press, Inc., San Diego, CA, 1994.

[9] Madore J., An introduction to noncommutative differential geometry and its physical applications, 2nd ed., London Mathematical Society Lecture Note Series, Vol. 257, Cambridge University Press, Cambridge, 1999.

[10] Connes A., Lott J., Particle models and noncommutative geometry, Nuclear Phys. B Proc. Suppl. 18 (1991), 29-47.

Chamseddine A.H., Connes A., The spectral action principle, Comm. Math. Phys. 186 (1997), 731-750, hep-th/9606001.

Chamseddine A.H., Connes A., Conceptual explanation for the algebra in the noncommutative approach to the standard model, Phys. Rev. Lett. 99 (2007), 191601, 4 pages, arXiv:0706.3690.

[11] Martín C.P., Gracia-Bondía M.J., Várilly J.C., The standard model as a noncommutative geometry: the low-energy regime, Phys. Rep. 294 (1998), 363-406, hep-th/9605001.

[12] Dubois-Violette M., Madore J., Kerner R., Gauge bosons in a noncommutative geometry, Phys. Lett. B 217 (1989), 485-488.

Dubois-Violette M., Madore J., Kerner R., Classical bosons in a noncommutative geometry, Classical Quantum Gravity 6 (1989), 1709-1724.

Dubois-Violette M., Kerner R., Madore J., Noncommutative differential geometry and new models of gauge theory, J. Math. Phys. 31 (1990), 323-330.

[13] Madore J., On a quark-lepton duality, Phys. Lett. B 305 (1993), 84-89.

Madore J., On a noncommutative extension of electrodynamics, in Spinors, Twistors, Clifford Algebras and Quantum Deformations (Sobotka Castle, 1992), Fund. Theories Phys., Vol. 52, Kluwer Acad. Publ., Dordrecht, 1993, 285-298. hep-ph/9209226.

[14] Connes A., Douglas M.R., Schwarz A., Non-commutative geometry and matrix theory: compactification on tori, J. High Energy Phys. 1998 (1998), no. 2, 003, 35 pages, hep-th/9711162.

[15] Seiberg N., Witten E., String theory and noncommutative geometry, J. High Energy Phys. 1999 (1999), no. 9, 032, 93 pages, hep-th/9908142.

[16] Chaichian M., Prešnajder P., Sheikh-Jabbari M.M., Tureanu A., Non-commutative standard model: model building, Eur. Phys. J. C 29 (2003), 413-432, hep-th/0107055.

[17] Jurčo B., Schraml S., Schupp P., Wess J., Enveloping algebra-valued gauge transformations for non-Abelian gauge groups on non-commutative spaces, Eur. Phys. J. C 17 (2000), 521-526, hep-th/0006246.

Jurčo B., Schupp P., Wess J., Nonabelian noncommutative gauge theory via noncommutative extra dimensions, Nuclear Phys. B 604 (2001), 148-180, hep-th/0102129. 
Jurčo B., Möller L., Schraml S., Schupp S., Wess J., Construction of non-Abelian gauge theories on noncommutative spaces, Eur. Phys. J. C 21 (2001), 383-388, hep-th/0104153.

Barnich G., Brandt F., Grigoriev M., Seiberg-Witten maps and noncommutative Yang-Mills theories for arbitrary gauge groups, J. High Energy Phys. 2002 (2002), no. 8, 023, 13 pages, hep-th/0206003.

[18] Calmet X., Jurčo B., Schupp P., Wess J., Wohlgenannt M., The standard model on non-commutative spacetime, Eur. Phys. J. C 23 (2002), 363-376, hep-ph/0111115.

Aschieri P., Jurčo B., Schupp P., Wess J., Non-commutative GUTs, standard model and $C, P, T$, Nuclear Phys. B 651 (2003), 45-70, hep-th/0205214.

[19] Aschieri P., Madore J., Manousselis P., Zoupanos G., Dimensional reduction over fuzzy coset spaces, J. High Energy Phys. 2004 (2004), no. 4, 034, 24 pages, hep-th/0310072.

Aschieri P., Madore J., Manousselis P., Zoupanos G., Unified theories from fuzzy extra dimensions, Fortschr. Phys. 52 (2004), 718-723, hep-th/0401200.

Aschieri P., Madore J., Manousselis P., Zoupanos G., Renormalizable theories from fuzzy higher dimensions, in Proceedings of the 3rd Summer School in Modern Mathematical Physics, Editors B. Dragovich, Z. Rakic and B. Sazdovic, Institute of Physics, Belgrade, 2005, 135-146, hep-th/0503039.

[20] Aschieri P., Grammatikopoulos T., Steinacker H., Zoupanos G., Dynamical generation of fuzzy extra dimensions, dimensional reduction and symmetry breaking, J. High Energy Phys. 2006 (2006), no. 9, 026, 26 pages, hep-th/0606021.

Aschieri P., Steinacker H., Madore J., Manousselis P., Zoupanos G., Fuzzy extra dimensions: dimensional reduction, dynamical generation and renormalizability, in Proceedings of the 4th Summer School in Modern Mathematical Physics, Editors B. Dragovich and Z. Rakic, Institute of Physics, Belgrade, 2007, 25-42, arXiv:0704.2880.

[21] Steinacker H., Zoupanos G., Fermions on spontaneously generated spherical extra dimensions, J. High Energy Phys. 2007 (2007), no. 9, 017, 35 pages, arXiv:0706.0398.

[22] Chatzistavrakidis A., Steinacker H., Zoupanos G., On the fermion spectrum of spontaneously generated fuzzy extra dimensions with fluxes, Fortschr. Phys. 58 (2010), 537-552, arXiv:0909.5559.

[23] Chatzistavrakidis A., Steinacker H., Zoupanos G., Orbifolds, fuzzy spheres and chiral fermions, J. High Energy Phys. 2010 (2010), no. 5, 100, 27 pages, arXiv:1002.2606.

[24] Madore J., The fuzzy sphere, Classical Quantum Gravity 9 (1992), 69-87.

[25] Balachandran A.P., Dolan B.P., Lee J.H., X. Martin X., O'Connor D., Fuzzy complex projective spaces and their star-products, J. Geom. Phys. 43 (2002), 184-204, hep-th/0107099.

Carow-Watamura U., Steinacker H., Watamura S., Monopole bundles over fuzzy complex projective spaces, J. Geom. Phys. 54 (2005), 373-399, hep-th/0404130.

Dolan B.P., Huet I., Murray S., O'Connor D., A universal Dirac operator and noncommutative spin bundles over fuzzy complex projective spaces, J. High Energy Phys. 2008 (2008), no. 3, 029, 21 pages, arXiv:0711.1347.

[26] Trivedi S.P., Vaidya S., Fuzzy cosets and their gravity duals, J. High Energy Phys. 2000 (2000), no. 9, 041, 33 pages, hep-th/0007011.

Dolan B.P., Jahn O., Fuzzy complex Grassmannian spaces and their star products, Internat. J. Modern Phys. A 18 (2003), 1935-1958, hep-th/0111020.

[27] Madore J., Schraml S., Schupp P., Wess J., Gauge theory on noncommutative spaces, Eur. Phys. J. C 16 (2000) 161-167, hep-th/0001203.

[28] Lechtenfeld O., Popov A.D., Szabo R.J., Rank two quiver gauge theory, graded connections and noncommutative vortices, J. High Energy Phys. 2006 (2006), no. 9, 054, 46 pages, hep-th/0603232.

Lechtenfeld O., Popov A.D., Szabo R.J., SU(3)-equivariant quiver gauge theories and nonabelian vortices, J. High Energy Phys. 2008 (2008), no. 8, 093, 63 pages, arXiv:0806.2791.

Dolan B.P., Szabo R.J., Dimensional reduction, monopoles and dynamical symmetry breaking, J. High Energy Phys. 2009 (2009), no. 3, 059, 27 pages, arXiv:0901.2491.

Dolan B.P., Szabo R.J., Equivariant dimensional reduction and quiver gauge theories, Gen. Relativity Gravitation, to appear, arXiv:1001.2429.

[29] Harland D., Kurkçuoğlu S., Equivariant reduction of Yang-Mills theory over the fuzzy sphere and the emergent vortices, Nuclear Phys. B 821 (2009), 380-398, arXiv:0905.2338.

[30] Barnes K.J., Forgacs P., Surridge M., Zoupanos G., On fermion masses in a dimensional reduction scheme, Z. Phys. C 33 (1987), 427-431.

[31] Steinacker H., Quantized gauge theory on the fuzzy sphere as random matrix model, Nuclear Phys. B 679 (2004), 66-98, hep-th/0307075. 
[32] Steinacker H., Gauge theory on the fuzzy sphere and random matrices, in Particle Physics and the Universe, Springer Proceedings in Physics, Vol. 98, Springer, Berlin, 2005, 307-311, hep-th/0409235.

[33] Carow-Watamura U., Watamura S., Noncommutative geometry and gauge theory on fuzzy sphere, Comm. Math. Phys. 212 (2000), 395-413, hep-th/9801195.

[34] Prešnajder P., Gauge fields on the fuzzy sphere, Modern Phys. Lett. A 18 (2003), 2431-2438.

[35] Andrews R.P., Dorey N., Spherical deconstruction, Phys. Lett. B 631 (2005), 74-82, hep-th/0505107. Andrews R.P., Dorey N., Deconstruction of the Maldacena-Núñez compactification, Nuclear Phys. B 751 (2006), 304-341, hep-th/0601098.

[36] Azuma T., Nagao K., Nishimura J., Perturbative dynamics of fuzzy spheres at large N, J. High Energy Phys. 2005 (2005), no. 6, 081, 21 pages, hep-th/0410263.

[37] Azuma T., Bal S., Nishimura J., Dynamical generation of gauge groups in the massive Yang-Mills-ChernSimons matrix model, Phys. Rev. D 72 (2005), 066005, 5 pages, hep-th/0504217.

[38] Azuma T., Bal S., Nagao K., Nishimura J., Nonperturbative studies of fuzzy spheres in a matrix model with the Chern-Simons term, J. High Energy Phys. 2004 (2004), no. 5, 005, 36 pages, hep-th/0401038.

[39] Aoki H., Iso S., Maeda T., Nagao K., Dynamical generation of a nontrivial index on the fuzzy 2-sphere, Phys. Rev. D 71 (2005), 045017, 10 pages, Erratum, Phys. Rev. D 71 (2005), 069905, 1 pages, hep-th/0412052.

[40] Aoki H., Nishimura J., Susaki Y., Suppression of topologically nontrivial sectors in gauge theory on $2 \mathrm{~d}$ non-commutative geometry, J. High Energy Phys. 2007 (2007), no. 10, 024, 17 pages, hep-th/0604093.

[41] Abel S.A., Jaeckel J., Khoze V.V., Ringwald A., Noncommutativity, extra dimensions, and power law running in the infrared, J. High Energy Phys. 2006 (2006), no. 1, 105, 22 pages, hep-ph/0511197.

[42] Lim C.S., Maru N., Hasegawa K., Six dimensional Gauge-Higgs unification with an extra space $S^{2}$ and the hierarchy problem, J. Phys. Soc. Japan 77 (2008), 074101, 15 pages, hep-th/0605180.

[43] Dvali G.R., Randjbar-Daemi S., Tabbash R., The origin of spontaneous symmetry breaking in theories with large extra dimensions, Phys. Rev. D 65 (2002), 064021, 13 pages, hep-ph/0102307.

[44] Antoniadis I., Benakli K., Quiros M., Supersymmetry and electroweak breaking by extra dimensions, Acta Phys. Polon. B 33 (2002), 2477-2488.

[45] Scrucca C.A., Serone M., Silvestrini L., Electroweak symmetry breaking and fermion masses from extra dimensions, Nuclear Phys. B 669 (2003), 128-158, hep-ph/0304220.

[46] Grosse H., Prešnajder P., The Dirac operator on the fuzzy sphere, Lett. Math. Phys. 33 (1995), $171-181$.

[47] Behr W., Meyer F., Steinacker H., Gauge theory on fuzzy $S^{2} \times S^{2}$ and regularization on noncommutative $\mathbb{R}^{4}$, J. High Energy Phys. 0507 (2005), no. 7, 040, 38 pages, hep-th/0503041.

[48] Maalampi J., Roos M., Physics of mirror fermions, Phys. Rep. 186 (1990), 53-96.

[49] Brink L., Schwarz J.H., Scherk J., Supersymmetric Yang-Mills theories, Nuclear Phys. B 121 (1977), 77-92. Gliozzi F., Scherk J., Olive D.I., Supersymmetry, supergravity theories and the dual spinor model, Nuclear Phys. B 122 (1977), 253-290.

[50] Candelas P., Horowitz G.T., Strominger A., Witten E., Vacuum configurations for superstrings, Nuclear Phys. B 258 (1985), 46-74.

[51] Cardoso G.L., Curio G., Dall'Agata G., Lüst D., Manousselis P., Zoupanos G., Non-Kähler string backgrounds and their five torsion classes, Nuclear Phys. B 652 (2003) 5-34, hep-th/0211118.

[52] Gauntlett J.P., Martelli D., Waldram D., Superstrings with intrinsic torsion, Phys. Rev. D 69 (2004), 086002 , 27 pages, hep-th/0302158.

[53] Dixon L.J., Harvey J.A., Vafa C., Witten E., Strings on orbifolds, Nuclear Phys. B 261 (1985), 678-686. Dixon L.J., Harvey J.A., Vafa C., Witten E., Strings on orbifolds. II, Nuclear Phys. B 274 (1986), $285-314$.

[54] Bailin D., Love A., Orbifold compactifications of string theory, Phys. Rep. 315 (1999), 285-408.

[55] Kachru S., Silverstein E., 4D conformal theories and strings on orbifolds, Phys. Rev. Lett. 80 (1998), 48554858, hep-th/9802183.

[56] Maldacena J.M., The large $N$ limit of superconformal field theories and supergravity, Adv. Theor. Math. Phys. 2 (1998), 231-252, hep-th/9711200.

Maldacena J.M., The large- $N$ limit of superconformal field theories and supergravity, Internat. J. Theoret. Phys. 38 (1999), 1113-1133.

[57] Douglas M.R., Moore G.W., D-branes, quivers, and ALE instantons, hep-th/9603167. 
[58] Douglas M.R., Greene B.R., Morrison D.R., Orbifold resolution by D-branes, Nuclear Phys. B 506 (1997), 84-106, hep-th/9704151.

[59] Aldazabal G., Ibáñez L.E., Quevedo F., Uranga A.M., D-branes at singularities: a bottom-up approach to the string embedding of the standard model, J. High Energy Phys. 2000 (2000), no. 8, 002, 70 pages, hep-th/0005067.

[60] Lawrence A.E., Nekrasov N., Vafa C., On conformal field theories in four dimensions, Nuclear Phys. B 533 (1998), 199-209, hep-th/9803015.

[61] Kiritsis E., D-branes in standard model building, gravity and cosmology, Phys. Rep. 421 (2005), 105-190, Erratum, Phys. Rep. 429 (2006), 121-122, hep-th/0310001.

[62] Djouadi A., The anatomy of electro-weak symmetry breaking. II. The Higgs bosons in the minimal supersymmetric model, Phys. Rep. 459 (2008), 1-241, hep-ph/0503173.

[63] Pati J.C., Salam A., Lepton number as the fourth "color", Phys. Rev. D 10 (1974), 275-289, Erratum, Phys. Rev. D 11 (1975), 703-703.

[64] Antoniadis I., Leontaris G.K., A supersymmetric SU(4) × O(4) model, Phys. Lett. B 216 (1989), 333-335. Antoniadis I., Leontaris G.K., Rizos J., A three-generation SU(4) × O(4) string model, Phys. Lett. B 245 (1990), 161-168.

[65] Anastasopoulos P., Leontaris G.K., Vlachos N.D., Phenomenological analysis of D-brane Pati-Salam vacua, J. High Energy Phys. 2010 (2010), no. 5, 011, 44 pages, arXiv:1002.2937.

[66] Ibanez L.E., A chiral $D=4, N=1$ string vacuum with a finite low energy effective field theory, J. High Energy Phys. 1998 (1998), no. 7, 002, 10 pages, hep-th/9802103.

[67] Ma E., Mondragón M., Zoupanos G., Finite $S U(N)^{k}$ unification, J. High Energy Phys. 2004 (2004), no. 12, 026, 17 pages, hep-ph/0407236.

[68] Glashow S.L., Trinification of all elementary particle forces, in Fifth Workshop on Grand Unification, Editors K. Kang, H. Fried and P. Frampton, World Scientific, Singapore, 1984, 88-94.

[69] Rizov V.A., A gauge model of the electroweak and strong interactions based on the group $\mathrm{SU}(3)_{L} \times \mathrm{SU}(3)_{R} \times$ $\mathrm{SU}(3)_{C}$, Bulg. J. Phys. 8 (1981), 461-477.

[70] Lazarides G., Panagiotakopoulos C., MSSM from SUSY trinification, Phys. Lett. B 336 (1994), 190-193, hep-ph/9403317.

[71] Heinemeyer S., Ma E., Mondragon M., Zoupanos G., Finite SU(3) ${ }^{3}$ model, AIP Conf. Proc. 1200 (2010) 568-571, arXiv:0910.0501.

[72] Babu K.S., He X.G., Pakvasa S., Neutrino masses and proton decay modes in $\mathrm{SU}(3) \times \mathrm{SU}(3) \times \mathrm{SU}(3)$ trinification, Phys. Rev. D 33 (1986), 763-772.

[73] Leontaris G.K., Rizos J., A D-brane inspired $\mathrm{U}(3)_{C} \times \mathrm{U}(3)_{L} \times \mathrm{U}(3)_{R}$ model, Phys. Lett. B 632 (2006), 710-716, hep-ph/0510230.

[74] Kim J.E., $Z_{3}$ orbifold construction of $\mathrm{SU}(3)^{3}$ GUT with $\sin ^{2}\left(\theta_{W}^{0}\right)=3 / 8$, Phys. Lett. B 564 (2003), 35-41, hep-th/0301177.

Choi K.S., Kim J.E., Three family $Z_{3}$ orbifold trinification, MSSM and doublet-triplet splitting problem, Phys. Lett. B $\mathbf{5 6 7}$ (2003), 87-92, hep-ph/0305002.

[75] Dienes K.R., Dudas E., Gherghetta T., Extra spacetime dimensions and unification, Phys. Lett. B 436 (1998), 55-65, hep-ph/9803466.

[76] Ghilencea D., Ross G.G., Unification and extra space-time dimensions, Phys. Lett. B 442 (1998), 165-172, hep-ph/9809217.

[77] Kobayashi T., Kubo J., Mondragon M., Zoupanos G., Running of soft parameters in extra space-time dimensions, Nuclear Phys. B 550 (1999) 99-122, hep-ph/9812221.

[78] Kubo J., Terao H., Zoupanos G., Kaluza-Klein thresholds and regularization (in)dependence, Nuclear Phys. B 574 (2000), 495-524, hep-ph/9910277.

[79] Filk T., Divergencies in a field theory on quantum space, Phys. Lett. B 376 (1996), 53-58.

Várilly J.C., Gracia-Bondía J.M., On the ultraviolet behaviour of quantum fields over noncommutative manifolds, Internat. J. Modern Phys. A 14 (1999), 1305-1323, hep-th/9804001.

Chaichian M., Demichev A., Prešnajder P., Quantum field theory on non-commutative space-times and the persistence of ultraviolet divergences, Nuclear Phys. B 567 (2000), 360-390, hep-th/9812180.

[80] Minwalla S., Van Raamsdonk M., Seiberg N., Noncommutative perturbative dynamics, J. High Energy Phys. 2000 (2000), no. 2, 020, 31 pages, hep-th/9912072. 
[81] Grosse H., Wulkenhaar R., Renormalisation of $\phi^{4}$-theory on non-commutative $\mathbb{R}^{4}$ to all orders, Lett. Math. Phys. 71 (2005), 13-26.

[82] Grosse H., Steinacker H., Finite gauge theory on fuzzy $\mathbb{C P}^{2}$, Nuclear Phys. B 707 (2005), 145-198, hep-th/0407089.

[83] Heinemeyer S., Mondragón M., Zoupanos G., Finite unification: theory and predictions, SIGMA 6 (2010), 049, 29 pages, arXiv:1001.0428.

[84] Ishibashi N., Kawai H., Kitazawa Y., Tsuchiya A., A large- $N$ reduced model as superstring, Nuclear Phys. B 498 (1997), 467-491, hep-th/9612115.

[85] Aoki H., Iso S., Suyama T., Orbifold matrix model, Nuclear Phys. B 634 (2002), 71-89, hep-th/0203277.

[86] Manousselis P., Zoupanos G., Supersymmetry breaking by dimensional reduction over coset spaces, Phys. Lett. B 504 (2001), 122-130, hep-ph/0010141.

Manousselis P., Zoupanos G., Soft supersymmetry breaking due to dimensional reduction over nonsymmetric coset spaces, Phys. Lett. B $\mathbf{5 1 8}$ (2001), 171-180, hep-ph/0106033.

Manousselis P., Zoupanos G., Dimensional reduction over coset spaces and supersymmetry breaking, J. High Energy Phys. 2002 (2002), no. 3, 002, 32 pages, hep-ph/0111125.

Manousselis P., Zoupanos G., Dimensional reduction of ten-dimensional supersymmetric gauge theories in the $N=1, D=4$ superfield formalism, J. High Energy Phys. 2004 (2004), no. 11, 025, 23 pages, hep$\mathrm{ph} / 0406207$.

[87] Chatzistavrakidis A., Manousselis P., Zoupanos G., Reducing the Heterotic Supergravity on nearly-Kähler coset spaces, Fortschr. Phys. 57 (2009), 527-534, arXiv:0811.2182.

Chatzistavrakidis A., Zoupanos G., Dimensional reduction of the heterotic string over nearly-Kähler manifolds, J. High Energy Phys. 2009 (2009), no. 9, 077, 39 pages, arXiv:0905.2398. 